\title{
APPLICATIONS OF GENERALIZED STRESS IN ELASTODYNAMICS
}

\author{
BY
}

\author{
DOUGLAS B. MEADE
}

Purdue University, West Lafayette, Indiana

\begin{abstract}
The problem under consideration is the scattering of elastic waves by inhomogeneous obstacles. The main goal is to obtain approximation techniques which are amenable to numerical implementation. For time-periodic problems a coupling procedure involving finite elements and boundary integral equations is described. For general time-dependent problems, artificial boundary methods are studied. In both cases the concept of generalized stress, as originated by Kupradze, plays a central role. The analysis is restricted to planar two-dimensional problems since these illustrate the essential ideas.
\end{abstract}

1. Introduction. We treat the problem of scattering of elastic waves in an infinite homogeneous medium by inhomogeneous obstacles. The special case of twodimensional anti-plane strain problems was considered in [2] and [3]. This situation reduces to a scalar problem. We consider the more complicated problem of two-dimensional plane strain. Here one has vector problems with all the essential difficulties of the fully three-dimensional problem. (The latter could also be treated by the methods given here.)

To obtain a mathematical formulation of the planar interface problem, let $\Omega$ and $\Omega_{+}$be two (uniformly) isotropic, linearly elastic bodies which fill all of $\mathbb{R}^{2}$. The body $\Omega$ is bounded and composed of an inhomogeneous material with density $\rho_{-}$ and Lamé moduli $\mu_{-}$and $\lambda_{-}$, while the unbounded homogeneous body $\Omega_{+}$has density $\rho$ and Lamé moduli $\mu_{+}$and $\lambda_{+}$. The interface is $\Gamma:=\partial \Omega$, the boundary of $\Omega$.

Since the materials are linearly elastic and isotropic, the fields are the planar displacement

$$
W=W_{1}\left(x_{1}, x_{2}, t\right) e_{1}+W_{2}\left(x_{1}, x_{2}, t\right) e_{2},
$$

the infinitesimal strain tensor

$$
E:=\frac{1}{2}\left(\nabla W+\nabla W^{\top}\right),
$$

Received February 19, 1990.

1980 Mathematics Subject Classification (1985 Revision). Primary 65N30, 73C60.

Key words and phrases. Elasticity, generalized stress, interface problem, boundary integral equation, artificial boundary conditions.

(C)1991 Brown University 
and the Cauchy stress tensor

$$
\Sigma[W]:=2 \mu(x) E+\lambda(x)(\operatorname{tr} E) I .
$$

Positivity of the stress tensor is ensured by assuming the Lamé moduli satisfy $\mu>0$ and $3 \lambda+2 \mu>0$ pointwise. The normal component of the stress is the traction

$$
\Sigma^{n}[W]:=\Sigma[W] n \text {. }
$$

Assuming there are no body forces, the equation of motion is

$$
\rho \ddot{W}=\nabla \cdot \Sigma[W] .
$$

The two bodies are assumed to be in rigid contact. That is, the displacement and traction are continuous across the interface. The system is set in motion by an incident field $U^{0}$ in $\Omega_{+}$which satisfies the homogeneous equation of motion in all of $\mathbb{R}^{3}$. The complete formulation of the interface problem is most conveniently stated in terms of

$$
U:= \begin{cases}W, & x \in \Omega, \\ W-U^{0}, & x \in \Omega_{+},\end{cases}
$$

the total wave in $\Omega$ and the scattered wave in $\Omega$.

Problem 1.1. Given the incident plane wave $U^{0}: \mathbb{R}^{2} \times(0, \infty) \rightarrow \mathbb{R}^{2}$, determine the outgoing plane wave $U:\left(\Omega \cup \Omega_{+}\right) \times(0, \infty) \rightarrow \mathbb{R}^{2}$ such that

$$
\begin{array}{cl}
\rho \ddot{U}=\nabla \cdot \Sigma[U] & \text { in }\left(\Omega \cup \Omega_{+}\right) \times(0, \infty), \\
{[U]=U^{0},\left[\sum^{n}[U]\right]=\sum^{n}\left[U^{0}\right]^{+}} & \text {on } \Gamma \times(0, \infty), \\
U(\cdot, 0)=0, \dot{U}(\cdot, 0)=0 & \text { in } \Omega \cup \Omega_{+} .
\end{array}
$$

The outgoing condition guarantees the uniqueness of solutions. An energy argument shows that a sufficient condition for the solution to be outgoing is

$$
\lim _{r \rightarrow \infty} \int_{S_{r}}(\Sigma[U] \cdot \dot{U})(x, t)=0 \text { for all } t \in(0, \infty)
$$

where $S_{r}:=\left\{y \in \Omega_{+}:|y|=r\right\}$.

In an attempt to maintain simple, yet concise, notation, we adopt a composite notation for the density and Lamé moduli, e.g.,

$$
\rho(x):=\left\{\begin{array}{ll}
\rho_{-}(x), & x \in \Omega \\
\rho_{+}, & x \in \Omega_{+}
\end{array} .\right.
$$

In addition, all analysis is performed under the assumption that the material parameters and the interface are as smooth as is necessary.

REMARK. The anti-plane displacement is orthogonal to the cross-sections of the inclusion: $W=W_{3}\left(x_{1}, x_{2}, t\right) e_{3}$. The formulation of the corresponding interface problem is unchanged, but simplifies to a scalar problem for $W_{3}$ with the observation that $\Sigma[W]=\mu \nabla W$ and $\Sigma^{n}[W]$ is parallel to the normal derivative of $W$.

In Sec. 3 we study Problem 1.1 in the special case of steady-state, time-periodic motions and in Sec. 4 in the general time-dependent case. The essential tool in both cases is the generalized stress; this is discussed in Sec. 2. 
2. Generalized stress and traction. The original idea for using the generalized stress and traction in the study of elastic interface problems is found in the work of Kupradze [20]. These ideas are used in the present situation to construct integral representations for the exterior displacement (see Sec. 3.2) and stable artificial boundary conditions (see Sec. 4.3). The key observation in this development is that

$$
\nabla \cdot \Sigma[u]=\mu \Delta u+\alpha \nabla \cdot\left(\nabla u^{\top}\right)+(\lambda+\mu-\alpha) \nabla \cdot((\nabla \cdot u) I),
$$

for any constants $\alpha, \mu$, and $\lambda$.

DEFINITION 2.1. The generalized stress associated with the planar displacement $u$ is

$$
\Sigma^{\alpha}[u]:=\mu \nabla u+\alpha \nabla u^{\top}+(\lambda+\mu-\alpha)(\nabla \cdot u) I
$$

the corresponding generalized traction is $\Sigma^{n}[u]:=\Sigma^{\alpha}[v] n$.

The proof of the generalized Green's identities is a simple exercise in integration by parts (see [21]).

LEMMA 2.1. Let $P$ be a bounded region of $\mathbb{R}^{2}$ with a piecewise smooth boundary, $\partial P$, and let $\mu, \lambda$, and $\alpha$ be real-valued differentiable functions on $P$. For any complex-valued smooth functions $u, v$ the generalized first and second Green's identities are

$$
\begin{aligned}
\int_{P}(\nabla \cdot \Sigma[u]) \cdot \bar{v}= & \int_{\partial P} \Sigma^{n}[u] \cdot \bar{v}-\int_{P} \Sigma^{\alpha}[u] \cdot \nabla \bar{v} \\
& +\int_{P}\left(\nabla u^{\top}-(\nabla \cdot u) I\right) \nabla(\mu-\alpha) \cdot \bar{v}
\end{aligned}
$$

and

$$
\begin{aligned}
\int_{P}((\nabla \cdot \Sigma[u]) \cdot \bar{v}-u \cdot(\nabla \cdot \Sigma[\bar{v}]))= & \int_{\partial P}\left(\Sigma^{n} \alpha[u] \cdot \bar{v}-u \cdot \Sigma^{n}[\bar{v}]\right) \\
& +\int_{P}\left(\nabla u^{\top}-(\nabla \cdot u) I\right) \nabla(\mu-\alpha) \cdot \bar{v} \\
& -\int_{P} u \cdot\left(\nabla \bar{v}^{\top}-(\nabla \cdot \bar{v}) I\right) \nabla(\mu-\alpha) .
\end{aligned}
$$

Note that, in (2.1) and (2.2), the extraneous integrals over $P$ vanish whenever $\mu-\alpha$ is a constant. Moreover, the classical Green's identities are obtained when $\alpha=\mu$.

The central idea needed for our analysis is contained in the following result.

Lemma 2.2. There is a skew-symmetric $2 \times 2$ matrix $M$ for which

$$
\stackrel{n}{\Sigma}^{\alpha}[u]=\Sigma^{n}[u]+(\alpha-\mu) M u_{, \tau} .
$$

Moreover, $M$ is spatially constant on $\partial P$. (Here $u_{, \tau}$ is the tangential derivative of $u$ on $\partial P$.)

Proof. From the definitions of the classical and generalized stresses we have

$$
\begin{aligned}
\Sigma^{\alpha}[u] & =\mu \nabla u+\alpha \nabla u^{\top}+(\lambda+\mu-\alpha)(\nabla \cdot u) I \\
& =\Sigma[u]+(\alpha-\mu)\left(\nabla u^{\top}-(\nabla \cdot u) I\right)
\end{aligned}
$$


so that, on $\partial P$,

$$
\stackrel{n}{n}^{\alpha}[u]=\Sigma^{n}[u]+(\alpha-\mu)\left(\nabla u^{\top}-(\nabla \cdot u) I\right) n .
$$

To complete the proof introduce a local coordinate system in terms of the arclength and distance from $\partial P$ along normal lines. That is, let $\partial P=\{X(\tau): \tau$ arclength $\}$ and let $n(\tau)$ denote the outward unit normal at $X(\tau)$. Then $x=X(\tau)+\nu n(\tau)$ produces an orthogonal coordinate system, $(\tau, \nu)$, in a neighborhood of $\partial P$. For any smooth vector-valued function $u$ we know that

$$
\nabla u=\frac{u_{, \tau}}{Q(\tau, \nu)} \otimes X^{\prime}(\tau)+u_{, \nu} \otimes n(\tau)
$$

with $Q(\tau, \nu):=1+\nu \kappa(\tau)$ and curvature $\kappa(\tau)$. Hence

$$
M(\tau, \nu):=\frac{1}{Q(\tau, \nu)}\left(X^{\prime}(\tau) \otimes n(\tau)-n(\tau) \otimes X^{\prime}(\tau)\right) .
$$

The skew-symmetry of $M$ is immediate; the fact that $M(\cdot, 0)$ is constant is obtained with the use of the Frenet formulae.

3. Time-harmonic problem. One means of determining the large-time behavior of solutions to the time-dependent problem is to study the corresponding time-harmonic problem. In the current context, suppose $U$ is a solution to Problem 1.1 for a timeperiodic and monochromatic incident wave, i.e., $U^{0}(x, t)=\operatorname{Re}\left(u^{0}(x) e^{i \omega t}\right)$ where $\omega>0$ is the frequency. Solutions to Problem 1.1 ultimately have the same form: $U(x, t) \rightarrow \operatorname{Re}\left(u(x) e^{i \omega t}\right)$, as $t \rightarrow \infty$ (pointwise on $\mathbb{R}^{2}$ ). The complex-valued "amplitude" $u$ solves the following time-reduced interface problem.

Problem 3.1. Given $\omega>0$ and $u^{0}: \mathbb{R}^{2} \rightarrow \mathbb{C}^{2}$, determine $u: \Omega \cup \Omega_{+} \rightarrow \mathbb{C}^{2}$ which satisfies

$$
\begin{aligned}
\nabla \cdot \Sigma[u]+\rho \omega^{2} u=0 & \text { in } \Omega \cup \Omega_{+}, \\
{[u]=u^{0}, \quad[\Sigma[u]]=\Sigma\left[u^{0}\right]^{+} } & \text {on } \Gamma,
\end{aligned}
$$

and the elastic radiation conditions.

3.1. Uniqueness. The elastic radiation conditions in Problem 3.1 are the timeharmonic analogue of the outgoing condition of Problem 1.1. An explicit representation of these conditions is obtained from the Helmholtz Decomposition [22]. Thus, $u=u_{L}+u_{T}$ where $u_{L}$ and $u_{T}$, the longitudinal and transverse components of the displacement, satisfy

$$
\begin{array}{cc}
\Delta u_{L}=\left(\frac{\omega}{c_{L}}\right)^{2} \ddot{u}_{L}, & \Delta u_{T}=\left(\frac{\omega}{c_{T}}\right)^{2} \ddot{u}_{T}, \\
\nabla \times u_{L}=0, & \nabla \cdot u_{T}=0 .
\end{array}
$$

The constants $c_{L}:=\left(\left(\lambda_{+}+2 \mu_{+}\right) / \rho_{+}\right)^{1 / 2}$ and $c_{T}:=\left(\mu_{+} / \rho_{+}\right)^{1 / 2}$ are the irrotational (longitudinal) and equivoluminal (transverse) wave speeds. 
Definition 3.1. The displacement $u=u_{L}+u_{T}$ is said to satisfy the elastic radiation conditions if $u_{L}$ and $u_{T}$ satisfy the Sommerfeld radiation conditions:

$$
\begin{array}{lll}
\left|u_{L}\right|=\mathscr{O}\left(r^{-1 / 2}\right), & \left|\frac{\partial u_{L}}{\partial r}+i \frac{\omega}{c_{L}} u_{L}\right|=o\left(r^{-1 / 2}\right), \\
\left|u_{T}\right|=\mathscr{O}\left(r^{-1 / 2}\right), & \left|\frac{\partial u_{T}}{\partial r}+i \frac{\omega}{c_{T}} u_{T}\right|=o\left(r^{-1 / 2}\right) .
\end{array}
$$

The remainder of the uniqueness argument is standard [20, Chap. III]. An energy argument (with $u^{0}=0$ ) shows that

$$
\operatorname{Im} \int_{\Gamma_{r}} \Sigma^{n}[u] \cdot \bar{u}=0
$$

for all $r$. Then, utilizing the elastic radiation conditions, it is shown that

$$
0=o(1)-\frac{\omega}{c_{L}}\left(\lambda_{+}+2 \mu_{+}\right) \int_{\Gamma_{r}}\left|u_{L}\right|^{2}-\frac{\omega}{c_{T}} \mu_{+} \int_{\Gamma_{r}}\left|u_{T}\right|^{2} .
$$

Hence $\int_{\Gamma_{r}}\left|u_{L}\right|^{2}=o(1)$ and $\int_{\Gamma_{r}}\left|u_{L}\right|^{2}=o(1)$ and Rellich's Lemma [15, p. 109] implies that $u_{L}$ and $u_{T}$ are identically zero in $\Omega_{+}$. Further, the interface conditions imply that $u^{-}=0$ and ${ }^{n}[u]^{-}=0$ on $\Gamma$ and, by unique continuation, the solution must vanish in the interior. As a result, Problem 3.1 has at most one solution.

3.2. Integral representation. The reformulation of Problem 3.1 on a finite domain is based upon the potential theory for the time-harmonic Cauchy equation

$$
\mu_{+} \Delta u+\left(\lambda_{+}+\mu_{+}\right) \nabla \nabla \cdot u+\rho_{+} \omega^{2} u=0 .
$$

The fundamental solution of (3.3) is, in $\mathbb{R}^{2}$, the matrix-valued function (see [19])

$$
\mathscr{K}(x, \tilde{x})=-\frac{i}{4 \mu}\left(H_{0}^{(2)}\left(k_{T} r^{\prime}\right) I+\frac{1}{k_{T}^{2}} \nabla \nabla\left(H_{0}^{(2)}\left(k_{T} r^{\prime}\right)-H_{0}^{(2)}\left(k_{L} r^{\prime}\right)\right)\right)
$$

where $H_{0}^{(2)}$ is the zero-order Hankel function of the second kind, $k_{L}:=\omega / c_{L}, k_{T}:=$ $\omega / c_{T}$, and $r^{\prime}:=|x-\tilde{x}|$.

A family of integral representations for solutions of (3.3) is obtained from the generalized second Green's identity (2.2). The simple and generalized double layers for a density $\varphi$ are defined to be

$$
\begin{gathered}
\mathscr{S}[\varphi](\tilde{x}):=\int_{\Gamma} \mathscr{K}(x, \tilde{x}) \varphi(x) d s_{x}, \\
\mathscr{D}^{\alpha}[\varphi](\tilde{x}):=\int_{\Gamma} \Sigma^{n}[\mathscr{K}](x, \tilde{x}) \varphi(x) d s_{x},
\end{gathered}
$$

for all $\tilde{x} \in \Omega \cup \Omega_{+}$. (The kernel of the generalized double layer, $\Sigma^{n}{ }^{\alpha_{+}}[\mathscr{K}]$, is the matrix whose $j$ th column is $\Sigma^{n}+\left[\mathscr{K}^{j}\right]$, the generalized traction of the $j$ th column of $\mathscr{K}$. 
In addition to satisfying (3.3) in $\Omega$ and in $\Omega_{+}$and the elastic radiation conditions, $\mathscr{S}$ and $\mathscr{D}^{\alpha}$ have well-known jump relations [20]. Define, for $\tilde{x} \in \Gamma$, the boundary integral operators $S$ and $D^{\alpha+}$ by

$$
\begin{aligned}
S[\varphi](\tilde{x}) & :=\int_{\Gamma} \mathscr{K}(x, \tilde{x}) \varphi(x) d s_{x}, \\
D^{\alpha+}[\varphi](\tilde{x}) & :=\int_{\Gamma}^{n_{x} \alpha_{+}}[\mathscr{K}](x, \tilde{x}) \varphi(x) d s_{x} .
\end{aligned}
$$

Then, for $\tilde{x} \in \Gamma$,

$$
\begin{aligned}
\mathscr{S}[\varphi](\tilde{x})^{ \pm} & =S[\varphi](\tilde{x}), \\
\sum^{n}\left[\mathscr{D}^{\alpha}+[\varphi]\right](\tilde{x})^{ \pm} & =\Sigma^{\alpha}\left[D^{\alpha+}[\varphi]\right](\tilde{x}), \\
\mathscr{D}^{\alpha}+[\varphi](\tilde{x})^{ \pm} & =\mp \frac{1}{2} \varphi(\tilde{x})+D^{\alpha}+[\varphi](\tilde{x}) .
\end{aligned}
$$

THEOREM 3.1. (i) If $u$ is a solution to (3.3) in an interior region $\Omega$, then

$$
\begin{aligned}
& u=\mathscr{D}^{\alpha}{ }_{+}\left[u^{-}\right]-\mathscr{S}\left[\sum^{n} \alpha^{+}[u]^{-}\right] \quad \text { in } \Omega, \\
& \frac{1}{2} u^{-}=D^{\alpha_{+}}\left[u^{-}\right]-S\left[\sum^{\alpha_{+}}[u]^{-}\right] \quad \text { on } \Gamma \text {, }
\end{aligned}
$$

for any choice of the parameter $\alpha_{+}$.

(ii) If $u$ is a solution to (3.3) in an unbounded exterior region $\Omega_{+}$which satisfies the elastic radiation conditions, then

$$
\begin{aligned}
& u=\mathscr{S}\left[\sum^{n} \alpha^{\alpha_{+}}[u]^{+}\right]-\mathscr{D}^{\alpha_{+}}\left[u^{+}\right] \text {in } \Omega_{+}, \\
& \frac{1}{2} u^{+}=S\left[\sum^{n} \alpha_{+}[u]^{+}\right]-D^{\alpha_{+}}\left[u^{+}\right] \quad \text { on } \Gamma \text {, }
\end{aligned}
$$

for all constants $\alpha_{+}$.

3.3. Boundary integral reformulation. We use the direct method to develop equivalent problems on a finite domain. Suppose $u$ is a solution to Problem 3.1. Then, for any choice of the parameter $\alpha_{+}$,

$$
u=\mathscr{S}\left[\sum^{n} \alpha_{+}[u]^{+}\right]-\mathscr{D}^{\alpha_{+}}\left[u^{+}\right]
$$

in $\Omega_{+}$while, on $\Gamma$,

$$
\frac{1}{2} u^{+}=S\left[\Sigma^{n} \alpha_{+}[u]^{+}\right]-D^{\alpha_{+}}\left[u^{+}\right] .
$$

The goal is to eliminate all references to the exterior solution. This requires a jump relation for the generalized traction. Using Lemma 2.2 and (3.2) we obtain

$$
\left[\Sigma^{n}[u]\right]=\Sigma^{n}{ }^{\alpha}+\left[u^{0}\right]+[\alpha-\mu] M u_{, \tau}^{-}
$$

where $M$ is as defined in Lemma 2.2 and, for any $x \in \Gamma$, the jump in $\alpha-\mu$ across the interface is

$$
[\alpha-\mu](x):=\left(\alpha_{-}(x)-\mu_{-}(x)\right)-\left(\alpha_{+}-\mu_{+}\right) .
$$


The remaining occurrences of the exterior solution are eliminated from (3.8) with the use of the interface conditions, (3.2), and (3.9). This produces

$$
0=\frac{1}{2} u^{-}-S\left[\sum^{\alpha_{-}}[u]^{-}\right]+D^{\alpha_{+}}\left[u^{-}\right]+S\left[[\alpha-\mu] M u_{, \tau}^{-}\right]-u^{0} .
$$

Note that the assumption that $u^{0}$ satisfies the homogeneous equation in $\Omega$ implies that

$$
\frac{1}{2} u^{0}=D^{\alpha}+\left[u^{0}\right]-S\left[\Sigma^{\alpha}+\left[u^{0}\right]\right] \text { on } \Gamma \text {. }
$$

To complete the reformulation of Problem 3.1 we introduce the interior generalized traction as an auxiliary variable: $\Theta:=\sum^{n}{ }^{\alpha}-[u]^{-}$.

Problem $3.2_{\alpha}$. Given $\omega>0$ and $u^{0}$, determine the pair $(u, \Theta)$ such that

$$
\begin{aligned}
\nabla \cdot \Sigma[u]+\rho_{-} \omega^{2} u & =0 & & \text { in } \Omega, \\
\Sigma^{\alpha}-[u]^{-}-\Theta & =0 & & \text { on } \Gamma, \\
\frac{1}{2} u^{-}-S[\Theta]+D^{\alpha_{+}}\left[u^{-}\right]+S\left[[\alpha-\mu] M u_{, \tau}^{-}\right] & =u^{0} & & \text { on } \Gamma .
\end{aligned}
$$

REMARK. Similar reformulations can be obtained from a simple-layer ansatz. In this situation the auxiliary variable is the simple-layer potential. One benefit of this approach is that the solution in the exterior is more easily determined than by the direct method. The direct method is preferable in situations where the primary interest is the interfacial traction. The simple-layer process is described in [21].

REMARK. It is also observed in [3] that one can, in fact, form a family of problems depending on a parameter $\beta, 0 \leq \beta \leq 1$, for the anti-plane case. In particular when $\beta=\frac{1}{2}$ the resulting problem is symmetric, thus reducing the numerical complexity. A similar family can be obtained for the plane strain case (see [21]). Symmetric problems are also obtained from the coupling method presented in [5]. However, this method has the disadvantage that it requires the use of hypersingular kernels.

Theorem 3.2. Let $\alpha$ be given. Problems 3.1 and $3.2 \alpha$ are equivalent in the following sense:

(i) If $u$ is a solution to Problem 3.1, then $\left(\left.u\right|_{\Omega}, \Sigma^{n}{ }^{\alpha}-[u]^{-}\right)$solves Problem $3.2_{\alpha}$;

(ii) Assume $\rho_{-} \omega^{2}$ is not an eigenfrequency of the interior Dirichlet problem. If $(u, \Theta)$ is a (sufficiently smooth) solution of Problem $3.2_{\alpha}$ and the extension of $u$ to $\Omega_{+}$is

$$
u=\mathscr{S}\left[\Theta-[\alpha-\mu] M u_{, \tau}^{-}-\Sigma^{\alpha_{+}}\left[u^{0}\right]\right]-\mathscr{D}^{\alpha+}\left[u^{-}-u^{0}\right],
$$

then $u$ is a solution to Problem 3.1.

Proof. The argument leading to the formulation of Problem 3.2 verifies the claim in (i).

It is obvious that the extension to $\Omega_{+}$specified in (3.13) satisfies the exterior equation of motion and the elastic radiation conditions. It remains only to confirm 
that the interface conditions are satisfied. To verify $(3.2)_{1}$ we use (3.13) to compute $u^{+}$. Then (3.13), together with (3.6), yields

$$
\mathscr{S}\left[\Theta-[\alpha-\mu] M u_{, \tau}^{-}-\Sigma^{\alpha_{+}}\left[u^{0}\right]-\Sigma^{n} \alpha_{+}[u]^{+}\right]=0 .
$$

The hypothesis that $\rho_{-} \omega^{2}$ is not an eigenfrequency for the interior Dirichlet problem implies $S$ is injective [21]. Thus, recalling (3.9) and the definition of $\Theta$,

$$
0=\Theta-[\alpha-\mu] M u_{, \tau}^{-}-\Sigma^{n} \alpha_{+}\left[u^{0}\right]-\Sigma^{n} \alpha_{+}[u]^{+}=\left[\sum[u]\right]-\sum^{n}\left[U^{0}\right]^{+} .
$$

Therefore $(3.2)_{2}$ is satisfied and the extended $u$ is a solution of Problem 3.1.

3.4. Variational problems. Suppose $(u, \Theta)$ is a smooth solution to Problem $3.2_{\alpha}$. Multiplying (3.10) by a test function $\bar{v}$ and integrating over $\Omega$ produces, with the use of (3.11) and (2.2),

$$
\begin{aligned}
0= & -\int_{\Omega} \Sigma^{\alpha_{-}}[u] \cdot \nabla \bar{v}+\int_{\Omega}\left(\nabla u^{\top}-(\nabla \cdot u) I\right) \nabla\left(\mu_{-}-\alpha_{-}\right) \cdot \bar{v} \\
& +\int_{\Omega} \rho_{-} \omega^{2} u \cdot \bar{v}+\int_{\Gamma} \Theta \cdot \bar{v}^{-} .
\end{aligned}
$$

Next, let $\Psi$ be a test function on $\Gamma$. Multiply (3.12) by $2 \bar{\Psi}$ and integrate over $\Gamma$. This leads to

$$
\begin{aligned}
2 \int_{\Gamma} \bar{\Psi} \cdot u^{0}= & \int_{\Gamma} \bar{\Psi} \cdot u^{-}-2 \int_{\Gamma} \bar{\Psi} \cdot S[\Theta]+2 \int_{\Gamma} \bar{\Psi} \cdot D^{\alpha_{+}}\left[u^{-}\right] \\
& +2 \int_{\Gamma} \bar{\Psi} \cdot S\left[[\alpha-\mu] M u_{, \tau}^{-}\right] .
\end{aligned}
$$

We seek solutions in $\mathscr{H}:=H^{1}(\Omega) \times H^{-1 / 2}(\Gamma)$. Define $\mathscr{U}:=(u, \Theta)$ and $\mathscr{V}:=$ $(v, \Psi)$ and let $\langle\cdot, \cdot\rangle$ denote the $H^{-1 / 2}(\Gamma)-H^{1 / 2}(\Gamma)$ duality pairing.

Problem $3.3_{\alpha}$. Given $u^{0} \in H_{\text {loc }}^{1}\left(\mathbb{R}^{2}\right)$, find $\mathscr{U} \in \mathscr{H}$ such that $\mathscr{A}^{\alpha}(\mathscr{U}, \mathscr{V})=$ $\mathscr{F}(\mathscr{V})$ for all $\mathscr{V} \in \mathscr{H}$.

The functional $\mathscr{F}$ and bilinear form $\mathscr{A}^{\alpha}$ are defined by $\mathscr{F}(\mathscr{V}):=2\left\langle\bar{\Psi}, u^{0}\right\rangle$ and

$$
\mathscr{A}^{\alpha}(\mathscr{U}, \mathscr{V}):=\mathscr{A}_{11}^{\alpha}(u, v)+\mathscr{A}_{12}(\Theta, v)+\mathscr{A}_{21}^{\alpha}(u, \Psi)+\mathscr{A}_{22}(\Theta, \Psi)
$$

where

$$
\begin{aligned}
\mathscr{A}_{11}^{\alpha}(u, v):= & \int_{\Omega} \Sigma^{\alpha}-[u] \cdot \nabla \bar{v}-\int_{\Omega} \rho_{-} \omega^{2} u \cdot \bar{v} \\
& -\int_{\Omega}\left(\nabla u^{\top}-(\nabla \cdot u) I\right) \nabla\left(\mu_{-}-\alpha_{-}\right) \cdot \bar{v}, \\
\mathscr{A}_{12}(\Theta, v):= & -\left\langle\Theta, \bar{v}^{-}\right\rangle, \\
\mathscr{A}_{21}^{\alpha}(u, \Psi):= & \left\langle\bar{\Psi}, u^{-}+2 D^{\alpha_{+}}\left[u^{-}\right]+2 S\left[[\alpha-\mu] M u_{, \tau}^{-}\right]\right\rangle, \\
\mathscr{A}_{22}(\Theta, \Psi):= & -2\langle\bar{\Psi}, S[\Theta]\rangle .
\end{aligned}
$$

3.5. Existence. A Riesz-Schauder argument is used to show the existence of a solution to Problem 3.3. The idea is to separate the bilinear form $\mathscr{A}^{\alpha}$ into the 
sum of a coercive term (on $\mathscr{H}$ ) and a "smoothing" term. The use of the generalized traction is critical to the success of this procedure.

Let $(\cdot, \cdot)$ be the $\mathscr{H}^{\prime}-\mathscr{H}$ duality pairing and write

$$
\mathscr{A}^{\alpha}(\mathscr{U}, \mathscr{V})=\left(\mathscr{A}_{0}^{\alpha} \mathscr{U}, \mathscr{V}\right)+\left(\mathscr{A}_{1}^{\alpha} \mathscr{U}, \mathscr{V}\right)
$$

where the bounded linear operators $\mathscr{A}_{0}^{\alpha}, \mathscr{A}_{1}^{\alpha}: \mathscr{H} \rightarrow \mathscr{H}^{\prime}$ are defined by

$$
\begin{aligned}
\left(\mathscr{A}_{0}^{\alpha} \mathscr{U}, \mathscr{V}\right):= & \int_{\Omega} \Sigma^{\alpha_{-}}[u] \cdot \nabla \bar{v}+\int_{\Omega} u \cdot \bar{v}-\left\langle\Theta, \bar{v}^{-}\right\rangle+\left\langle\bar{\Psi}, u^{-}\right\rangle-2\langle\bar{\Psi}, \widetilde{S}[\Theta]\rangle, \\
\left(\mathscr{A}_{1}^{\alpha} \mathscr{U}, \mathscr{V}\right):= & -\int_{\Omega}\left(\rho_{-} \omega^{2}+1\right) u \cdot \bar{v}-\int_{\Omega}\left(\nabla u^{\top}-(\nabla \cdot u) I\right) \nabla\left(\mu_{-}-\alpha_{-}\right) \cdot \bar{v} \\
& +2\left\langle\bar{\Psi}, D^{\alpha_{+}}\left[u^{-}\right]\right\rangle+2\left\langle\bar{\Psi}, S\left[[\alpha-\mu] M u_{, \tau}^{-}\right]\right\rangle-2\langle\bar{\Psi},(S-\widetilde{S})[\Theta]\rangle,
\end{aligned}
$$

for all $\mathscr{U}, \mathscr{V} \in \mathscr{H}$. The boundary integral operator $\widetilde{S}$ is the trace of the simple layer $\widetilde{S}$ for the homogeneous elliptic equation

$$
-\mu_{+} \Delta u-\left(\mu_{+}+\lambda_{+}\right) \nabla \nabla \cdot u+u=0 .
$$

Observe that formally

$$
\begin{aligned}
& \mathscr{A}_{0}^{\alpha} \mathscr{U}=\left[\begin{array}{c}
-\nabla \cdot \Sigma^{\alpha}-[u]+u-\Theta \\
u^{-}-2 \widetilde{S}[\Theta]
\end{array}\right], \\
& \mathscr{A}_{1}^{\alpha} \mathscr{U}=\left[\begin{array}{c}
-\left(\rho_{-} \omega^{2}+1\right) u-\left(\nabla u^{\top}-(\nabla \cdot u) I\right) \nabla\left(\mu_{-}-\alpha_{-}\right) \\
2 D^{\alpha}+\left[u^{-}\right]+2 S\left[[\alpha-\mu] M u_{, \tau}^{-}\right]-2(S-\widetilde{S})[\Theta]
\end{array}\right],
\end{aligned}
$$

and Problem $3.3_{\alpha}$ can be rewritten as

$$
\mathscr{A}_{0}^{\alpha} \mathscr{U}+\mathscr{A}_{1}^{\alpha} \mathscr{U}=F:=\left[\begin{array}{c}
0 \\
2 u^{0}
\end{array}\right] .
$$

To investigate the coercivity of $\mathscr{A}_{0}^{\alpha}$ on $\mathscr{H}$, let $\mathscr{U}=(u, \Theta) \in \mathscr{H}$ be given. Then

$$
\operatorname{Re}\left(\mathscr{A}_{0}^{\alpha} \mathscr{U}, \mathscr{U}\right)=\int_{\Omega}\left(\Sigma^{\alpha}-[u] \cdot \nabla \bar{u}+|u|^{2}\right)-2 \operatorname{Re}\langle\bar{\Theta}, \tilde{S}[\Theta]\rangle .
$$

It suffices to show that the individual terms are coercive on $H^{1}(\Omega)$ and $H^{-1 / 2}(\Gamma)$, respectively.

Lemma 3.1. Suppose $\alpha_{-}$is selected so that $\alpha_{-} \in\left(-\mu_{-}, \mu_{-}\right]$on $\Omega$. Then there is a positive constant $C$ such that

$$
\int_{\Omega}\left(\Sigma^{\alpha}-[u] \cdot \nabla \bar{u}+|u|^{2}\right) \geq C\|u\|_{H^{1}(\Omega)}^{2}
$$

for all $u \in H^{1}(\Omega)$.

LemMA 3.2. The operator $\widetilde{S}$ is bijective from $H^{-1 / 2}(\Gamma)$ to $H^{1 / 2}(\Gamma)$ and coercive on $H^{-1 / 2}(\Gamma)$, i.e., there is a constant $C>0$ so that

$$
-\operatorname{Re}\langle\bar{\varphi}, \tilde{S}[\varphi]\rangle \geq C\|\varphi\|_{H^{-1 / 2}(\Gamma)}^{2}
$$

for all $\varphi \in H^{-1 / 2}(\Gamma)$. 
These results are obtained by a diligent investigation of the singularity of the kernel of $\widetilde{S}$ (see [18]). The complete proofs for the present context are in [21].

Proof of Lemma 3.1. From the definition of the generalized stress we find

$$
\begin{aligned}
\Sigma^{\alpha_{-}}[u] \cdot \nabla \bar{u}+|u|^{2}= & \left(\mu_{-}-\alpha_{-}\right)\left|\frac{\nabla u-\nabla u^{\top}}{2}\right|^{2}+\left(\mu_{-}+\alpha_{-}\right)\left|\frac{\nabla u+\nabla u^{\top}}{2}\right|^{2} \\
& +\left(\lambda_{-}+\mu_{-}-\alpha_{-}\right)|\nabla \cdot u|^{2}+|u|^{2} .
\end{aligned}
$$

Then, using the estimate

$$
(a E+b(\operatorname{tr} E) I) \cdot E \geq \min \{a, a+3 b\}|E|^{2}
$$

for symmetric tensors [14, p. 85] and applying Korn's Second Inequality [9], we conclude that, for all $u \in H^{1}(\Omega)$,

$$
\int_{\Omega}\left(\Sigma^{n}-[u] \cdot \nabla \bar{u}+|u|^{2}\right) \geq C \int_{\Omega}\left(|\nabla u|^{2}+|u|^{2}\right)
$$

provided

$$
\begin{gathered}
\mu_{-}+\alpha_{-} \geq 0, \\
\left(\mu_{-}+\alpha_{-}\right)+3\left(\lambda_{-}+\mu_{-}-\alpha_{-}\right) \geq 0, \\
\mu_{-}-\alpha_{-} \geq 0 .
\end{gathered}
$$

Combining all three restrictions, (3.15) holds for any $\alpha_{-} \in\left(-\mu_{-}, \mu_{-}\right]$.

The coercivity of $\mathscr{A}_{0}^{\alpha}$ implies the existence of an inverse $T^{\alpha}: \mathscr{H}^{\prime} \rightarrow \mathscr{H}$. Applying $T^{\alpha}$ to $(3.14)$ yields

$$
\mathscr{U}+\left(T^{\alpha} \mathscr{A}_{1}^{\alpha}\right) \mathscr{U}=T^{\alpha} F .
$$

If $T^{\alpha} \mathscr{A}_{0}^{\alpha}$ is compact, as an operator on $\mathscr{H}$, then (3.16) is a Riesz-Schauder equation on $\mathscr{H}$. As such, a solution to Problem $3.3_{\alpha}$ exists if $\mathscr{U}=0$ is the unique solution to the homogeneous equation

$$
\mathscr{U}+\left(T^{\alpha} \mathscr{A}_{1}^{\alpha}\right) \mathscr{U}=0 .
$$

The compactness of $T^{\alpha} \mathscr{A}_{0}^{\alpha}$ and the unique solvability of (3.17) are directly related to the smoothing properties of $S-\widetilde{S}$ and $D^{\alpha_{+}}$.

LEMMA 3.3. (i) The difference $S-\widetilde{S}$ is a pseudo-differential operator of order -3 , i.e., $S-\widetilde{S}: H^{-1 / 2+\epsilon}(\Gamma) \rightarrow H^{5 / 2+\epsilon}(\Gamma)$ for any $\epsilon \geq 0$;

(ii) The smoothing of $D^{\alpha_{+}}$depends upon the choice of the constant $\alpha_{+}$. In general, $D^{\alpha_{+}}$is a pseudo-differential operator of order 0 . However, if

$$
\alpha_{+}=\tilde{\alpha}_{+}:=\frac{\mu_{+}\left(\mu_{+}+\lambda_{+}\right)}{\lambda_{+}+3 \mu_{+}},
$$

then the order of $D^{\alpha_{+}}$is -1 , and $D^{\alpha_{+}}: H^{1 / 2+\epsilon}(\Gamma) \rightarrow H^{3 / 2+\epsilon}(\Gamma)$.

Proof. The smoothness of these mappings is determined from asymptotic expansions of the kernels. The kernel of $\widetilde{S}$ is

$$
\widetilde{\mathscr{K}}(x, \tilde{x})=\frac{1}{2 \pi \mu}\left(K_{0}\left(\tilde{k}_{T}|x-\tilde{x}|\right) I+\frac{1}{\tilde{k}_{T}^{2}} \nabla \nabla\left(K_{0}\left(\tilde{k}_{T}|x-\tilde{x}|\right)-K_{0}\left(\tilde{k}_{L}|x-\tilde{x}|\right)\right)\right),
$$


where $K_{0}$ is the modified Bessel function of order zero, $\tilde{k}_{L}:=\left(\lambda_{+}+2 \mu_{+}\right)^{-1 / 2}$, and $\tilde{k}_{T}:=\mu_{+}^{-1 / 2}$. Small-amplitude expansions of $\mathscr{K}$ and $\widetilde{\mathscr{K}}$ show that

$$
(\mathscr{K}-\widetilde{\mathscr{K}})(x, \tilde{x})=C_{1}+C_{2}|x-\tilde{x}|^{2} \log |x-\tilde{x}|+\mathscr{O}\left(|x-\tilde{x}|^{2}\right) .
$$

To prove (i) we consider the kernels of the second-derivatives of $S-\widetilde{S}$. The singularities are $\mathscr{O}(\log |x-\tilde{x}|)$; hence one degree of smoothing is produced. It then follows that $S-\widetilde{S}$ is a pseudo-differential operator of order -3 .

The proof of the second half of the lemma is based upon Kupradze's derivation of the "pseudo-stress" [20, Sec. I.4]. The small-amplitude expansion of the kernel of $D^{\alpha_{+}}$is

$$
\begin{aligned}
\sum^{\alpha_{+}}[\mathscr{K}](x, \tilde{x})= & \left(b_{1} \mu_{+}+b_{2} \alpha_{+}\right)\left(\frac{\partial}{\partial n_{x}} \log |x-\tilde{x}|\right) I \\
& -2 b_{2}\left(\mu_{+}+\alpha_{+}\right)\left(\frac{\partial}{\partial n_{x}} \log |x-\tilde{x}|\right) \nabla_{x}|x-\tilde{x}| \otimes \nabla_{x}|x-\tilde{x}| \\
& +\left(b_{1} \alpha_{+}+b_{2} \mu_{+}\right) n \otimes \nabla_{x} \log |x-\tilde{x}| \\
& +\left(b_{1}\left(\lambda_{+}+\mu_{+}-\alpha_{+}\right)+b_{2}\left(\lambda_{+}+2 \mu_{+}\right)\right)\left(\nabla_{x} \log |x-\tilde{x}|\right) \otimes n \\
& +\mathscr{O}(|x-\tilde{x}| \log |x-\tilde{x}|)
\end{aligned}
$$

where $b_{1}:=-\left(\lambda_{+}+3 \mu_{+}\right) /\left(4 \pi \mu_{+}\left(\lambda_{+}+2 \mu_{+}\right)\right)$and $b_{2}:=\left(\lambda_{+}+\mu_{+}\right) /\left(4 \pi \mu_{+}\left(\lambda_{+}+2 \mu_{+}\right)\right)$. Note that the singularity of $\Sigma^{n} \alpha_{+}[\mathscr{K}]$ is, in general, a combination of normal and tangential derivatives of the logarithm. In these situations $D^{\alpha_{+}}$has a Cauchy kernel and provides no smoothing. However, if $\alpha_{+}$can be selected so that only the normal derivatives terms appear in (3.19), then one degree of smoothing will be obtained. The unique choice of the parameter $\alpha_{+}$for which this occurs is (3.18).

To complete the proof of the compactness of $T^{\alpha} \mathscr{A}_{1}^{\alpha}$, let $\alpha:=\tilde{\alpha}$ where $\tilde{\alpha}_{+}$is obtained from (3.18) and $\tilde{\alpha}_{-}$is defined so that $[\tilde{\alpha}-\mu]=0$. Then

$$
\mathscr{A}_{1}^{\dot{\alpha}}: H^{1+\epsilon}(\Omega) \times H^{-1 / 2+\epsilon}(\Gamma) \rightarrow H^{1+\epsilon}(\Omega) \times H^{3 / 2+\epsilon}(\Gamma)
$$

and, with $\mathscr{H}^{\epsilon}:=H^{1+\epsilon}(\Omega) \times H^{-1 / 2+\epsilon}(\Gamma)$,

$$
T^{\dot{\alpha}} \mathscr{A}_{1}^{\dot{\alpha}}: \mathscr{H}^{\epsilon} \rightarrow \mathscr{H}^{1+\epsilon}
$$

for any $\epsilon \geq 0$. Thus $T^{\grave{\alpha}} \mathscr{A}^{\grave{\alpha}_{1}}: \mathscr{H} \rightarrow \mathscr{H}^{1}$, and $T \mathscr{A}_{1}^{\grave{\alpha}}$ is compact as a mapping on $\mathscr{H}$.

To show that (3.17) has a unique solution, suppose $\mathscr{U} \in \mathscr{H}$ is one such solution. Then $\mathscr{U}$ is a solution to Problem $3.3_{\dot{\alpha}}$, with $u^{0}=0$, which is in $\mathscr{H}^{\epsilon}$ for every $\epsilon \geq 0$. In particular, $\mathscr{U}$ is smooth enough to invoke Theorem 3.2(ii). The uniqueness of solutions to Problem 3.1 implies that $\mathscr{U}=0$.

The existence of a solution to Problem $3.3_{\dot{\alpha}}$ is complete once we confirm that $\tilde{\alpha}$ satisfies all the hypotheses of Lemma 3.1, i.e., $\tilde{\alpha}_{-} \in\left(-\mu_{-}, \mu_{-}\right]$. That $\ddot{\alpha}<\mu_{-}$is easily determined from the assumptions that $\mu>0$ and $3 \lambda+2 \mu>0$. However, $\tilde{\alpha}_{-}>-\mu_{-}$only if $\mu_{-}>\mu_{+}^{2} /\left(\lambda_{+}+3 \mu_{+}\right)$. 
THEOREM 3.3. Define the function $\tilde{\alpha}$ by:

Assume

$$
\tilde{\alpha}(x):= \begin{cases}\mu_{-}(x)-\frac{2 \mu_{+}^{2}}{\lambda_{+}+3 \mu_{+}}, & x \in \Omega \\ \frac{\mu_{+}\left(\lambda_{+}+\mu_{+}\right)}{\lambda_{+}+3 \mu_{+}}, & x \in \Omega_{+} .\end{cases}
$$

$$
\mu_{-}>\frac{\mu_{+}^{2}}{\lambda_{+}+3 \mu_{+}} \text {in } \Omega
$$

and $\rho_{-} \omega^{2}$ is not an eigenfrequency of the interior Dirichlet problem. For every incident wave $u^{0} \in H_{\mathrm{loc}}^{1}\left(\mathbb{R}^{2}\right)$, Problem $3.3_{\dot{\alpha}}$ has a unique weak solution $\mathscr{U} \in \mathscr{H}$. Moreover, $\|\mathscr{U}\|_{\mathscr{H}} \leq C\left\|u^{0}\right\|_{H_{\mathrm{loc}}^{1}\left(\mathbb{R}^{2}\right)}$ for some constant $C>0$.

In practice, solutions to Problem $3.3_{\tilde{\alpha}}$ are sought via Galerkin's method. The existence proof just completed aiso suffices to establish quasi-optimal convergence of finite-dimensional solutions in $\mathscr{H}$ (see, e.g., $[6,17]$ ). Numerical implementation of these ideas is simplified by the observation that all boundary conditions are natural.

We conclude with some comments about the restriction on the interior shear modulus. Although many realistic combinations of materials do satisfy (3.20), for example a steel, aluminum alloy, and "unmodified" concrete foundation in the surface of the earth (see, e.g., [13]), there is no known physical justification for this constraint.

One explanation for the origin of this restriction is the lack of necessary conditions for the coercivity of $\mathscr{A}_{0}^{\alpha}$. In fact, Costabel and Stephan obtain existence results for any combination of (homogeneous) elastic materials [5]. It must be noted that the techniques in [5] require the use of integral operators with the hypersingular kernels; the methods which we have just completed utilize integral operators that are at most strongly singular. The author is continuing to examine several means of strengthening this part of the analysis.

4. Artificial boundary problems. The use of artificial boundaries for acoustic and elastic wave equations on two-dimensional half-spaces has been studied extensively (see, e.g., $[4,11,12,16]$ and the references therein). The basic approach is to use Laplace and Fourier transforms to obtain an explicit representation for the symbol of the artificial boundary condition. The exact boundary condition is nonlocal in both space and time; more localized approximate boundary conditions are obtained by truncating the Pade expansion of the symbol.

Recently, Barry, Bielak, and MacCamy [2] have generalized these methods to the scalar anti-plane problem in the exterior of a region with a curved boundary. The extension to the planar elastic interface problem is presented in this work.

4.1. Exact artificial boundary conditions. Let $U$ be a solution of Problem 1.1. Then $\hat{U}(x, \cdot)$, the Laplace transform of $U(x, \cdot)$, satisfies the elastic radiation conditions and

$$
\begin{aligned}
\rho s^{2} \widehat{U}=\nabla \cdot \Sigma[\widehat{U}] & \text { in } \Omega \cup \Omega_{+}, \\
{[\widehat{U}]=\widehat{U}^{0}, \quad\left[\Sigma^{n}[\widehat{U}]\right]=\Sigma\left[\widehat{U}^{0}\right]^{+} } & \text {on } \Gamma .
\end{aligned}
$$


The artificial boundary is $\Gamma_{1}$, a closed, convex, smooth curve surrounding $\Omega$. Define $\Omega_{1,+}$ to be the unbounded region with boundary $\Gamma_{1}$ and $\Omega_{1}:=\Omega_{+} \backslash \overline{\Omega_{1,+}}$. To obtain boundary conditions on the artificial boundary we consider a family of well-posed elliptic Dirichlet problems in $\Omega_{1,+}$.

Problem $4.1_{t}$. Determine the function $\hat{\mathscr{U}}(\cdot, \cdot ; t)$ which satisfies the elastic radiation conditions and

$$
\begin{aligned}
\rho s^{2} \hat{\mathscr{U}}(x, s ; t) & =\nabla \cdot \Sigma[\hat{\mathscr{U}}](x, s ; t) & & \text { in } \Omega_{1,+} \\
\hat{\mathscr{U}}(x, s ; t) & =U(x, t) & & \text { on } \Gamma_{1} .
\end{aligned}
$$

From classical elliptic existence theory there exists a bounded linear operator $\hat{\mathscr{G}}$ (independent of $t$ ) for which $\hat{\mathscr{U}}(x, s ; t)=\hat{\mathscr{G}}\left[\left.U\right|_{\Gamma_{1}}(\cdot, t)\right](x, s)$ in $\Omega_{1,+}$. Thus, for any $\alpha$, the generalized traction of $\hat{\mathscr{U}}$ on $\Gamma_{1}$ is given by

$$
\Sigma^{n}{ }^{\alpha}+\left.[\widehat{\mathscr{U}}]\right|_{\Gamma_{1}}(\cdot, \cdot ; t)=\widehat{\mathscr{F}}^{\alpha}\left[\left.U\right|_{\Gamma_{1}}(\cdot, t)\right],
$$

where $\widehat{\mathscr{F}}^{\alpha}$ is a bounded linear operator. The generalized traction of $\widehat{U}$ is found by applying the Laplace transform to (4.2):

$$
\begin{aligned}
\sum^{\alpha}+\left.[\widehat{U}]\right|_{\Gamma_{1}}(x, s) & =\int_{0}^{\infty} e^{-s t} \sum^{\alpha}+\left.[\widehat{\mathscr{U}}]\right|_{\Gamma_{1}}(x, s ; t) d t \\
& =\int_{0}^{\infty} e^{-s t} \widehat{\mathscr{F}}^{\alpha}\left[\left.U\right|_{\Gamma_{1}}(\cdot, t)\right](x, s) d t \\
& =\widehat{\mathscr{F}}^{\alpha}\left[\left.\widehat{U}\right|_{\Gamma_{1}}(\cdot, s)\right](x, s) .
\end{aligned}
$$

The corresponding artificial boundary condition for $U$ is, in the time domain,

$$
\sum^{n}{ }^{\alpha}+\left.[U]\right|_{\Gamma_{1}}(\cdot, t)=\mathscr{F}^{\alpha}\left[\left.U^{t}\right|_{\Gamma_{1}}\right]
$$

with $U^{t}(\cdot, \tau):=U(\cdot, t-\tau)$, the time history of $U$ at time $t$. The formulation of the exact artificial boundary problem is now complete.

Problem $4.2_{\alpha}$. Given $U^{0}$, find the planar wave $U$ that satisfies

$$
\begin{array}{cl}
\rho \ddot{U}=\nabla \cdot \Sigma[U] & \text { in }\left(\Omega \cup \Omega_{1}\right) \times(0, \infty), \\
\Sigma^{n} \alpha_{+}[U]=\mathscr{F}^{\alpha}\left[U^{t}\right] & \text { on } \Gamma_{1} \times(0, \infty), \\
{[U]=U^{0}, \quad\left[\sum^{n}[U]\right]=\Sigma^{n}\left[U^{0}\right]^{+}} & \text {on } \Gamma \times(0, \infty), \\
U(\cdot, 0)=0, \quad \dot{U}(\cdot, 0)=0 & \text { in } \Omega \cup \Omega_{1} .
\end{array}
$$

The impracticality of the exact artificial boundary condition is apparent. First, $\mathscr{F}^{\alpha}$ is defined solely through the abstract theory of elliptic PDEs and the Laplace transform. Further, even if an explicit definition of $\mathscr{F}^{\alpha}$ were available, the spatial and temporal nonlocality complicates any computational implementation.

4.2. Approximate artificial boundary conditions. Approximations to $\mathscr{F}^{\alpha}$ are obtained by truncating an asymptotic expansion of $\widehat{\mathscr{F}}^{\alpha}$. The expansion of $\widehat{\mathscr{F}}^{\alpha}$ is 
based upon the ideas of geometrical optics for wave equations [7]. The Helmholtz Decomposition permits the replacement of the exterior equation of motion (4.1) with a pair of (scalar) reduced wave equations in $\Omega_{1,+}$. That is

$$
\widehat{\mathscr{U}}=\nabla \widehat{\Phi}+\nabla \times\left(\widehat{\Phi} e_{3}\right)
$$

where

$$
\Delta \widehat{\Phi}=\left(\frac{s}{c_{L}}\right)^{2} \widehat{\Phi}, \quad \Delta \widehat{\Psi}=\left(\frac{s}{c_{T}}\right)^{2} \widehat{\Psi} .
$$

High-frequency $(s \gg 1)$ expansions of $\widehat{\Phi}$ and $\widehat{\Psi}$ are

$$
\begin{aligned}
& \widehat{\Phi}(x, s ; t) \sim e^{-s p(x)} \sum_{k=1}^{\infty} \Phi_{k}(x ; t) s^{-k}, \\
& \widehat{\Psi}(x, s ; t) \sim e^{-s q(x)} \sum_{k=1}^{\infty} \Psi_{k}(x ; t) s^{-k} .
\end{aligned}
$$

The computations simplify with a change of coordinates. Suppose $\Gamma_{1}=\{X(\tau): \tau$ arclength $\}$ and $n(\tau)$ is the outward unit normal at $X(\tau)$. Then $x=X(\tau)+\nu n(\tau)$ defines an orthogonal coordinate system $(\tau, \nu)$, where $\nu$ is the distance from $x$ to $\Gamma_{1}$ as measured along the normal $n(\tau)$. Let $Q=Q(\tau, \nu):=1+\nu \kappa(\tau)$ where $\kappa(\tau) \geq 0$ is the curvature at $X(\tau)$. For future reference we recall that for a scalarvalued function $\chi$ :

$$
\nabla \chi=\frac{\chi, \tau}{Q} X^{\prime}(\tau)+\chi_{, \nu} n(\tau), \quad \Delta \chi=\frac{1}{Q}\left(\left(\frac{\chi_{, \tau}}{Q}\right)_{, \tau}+\left(Q \chi_{, \nu}\right)_{, \nu}\right)
$$

and for a vector-valued function $v$ :

$$
\nabla v=\frac{v_{, \tau}}{Q} \otimes X^{\prime}(\tau)+v_{, \nu} \otimes n(\nu)
$$

where $\otimes$ denotes the tensor product.

The functions $p$ and $q$ are determined by substituting (4.5) and (4.6) into (4.4) and (4.2). Thus, for $\nu \geq 0$,

$$
|\nabla p|^{2}=\frac{1}{c_{L}^{2}}, \quad|\nabla q|^{2}=\frac{1}{c_{T}^{2}}
$$

while $p=0$ and $q=0$ when $\nu=0$. As a result

$$
p(x)=\frac{\nu}{c_{L}}, \quad q(x)=\frac{\nu}{c_{T}} .
$$

To express the generalized traction in the new coordinates we first decompose $\widehat{\mathscr{U}}$ into its tangential and normal components: $\widehat{\mathscr{U}}=\widehat{\mathscr{U}}^{T} X^{\prime}+\widehat{\mathscr{U}}^{N} n$ :

$$
\begin{aligned}
{\left.\stackrel{\Sigma}{\alpha^{\alpha}}[\hat{\mathscr{U}}]\right|_{\Gamma_{1}}=} & \left.\mu_{+}(\nabla \hat{\mathscr{U}})\right|_{\Gamma_{1}} n+\left.\alpha_{+}(\nabla \widehat{\mathscr{U}})^{\top}\right|_{\Gamma_{1}} n+\left.\left(\lambda_{+}+\mu_{+}-\alpha_{+}\right)(\nabla \cdot \hat{\mathscr{U}})\right|_{\Gamma_{1}} n \\
= & \left(\mu_{+} \hat{\mathscr{U}}_{, \nu}^{T}+\alpha_{+}\left(\hat{\mathscr{U}}_{\tau}^{N}-\kappa \hat{\mathscr{U}}^{T}\right)\right) X^{\prime} \\
& +\left(\left(\lambda_{+}+2 \mu_{+}\right) \hat{\mathscr{U}}_{, \nu}^{N}+\left(\lambda_{+}+\mu_{+}-\alpha_{+}\right)\left(\hat{\mathscr{U}}_{, \tau}^{T}+\kappa \widehat{\mathscr{U}}^{N}\right)\right) n .
\end{aligned}
$$


The asymptotic expansions of $\widehat{\mathscr{U}}^{T}$ and $\widehat{\mathscr{U}}^{N}$ on $\Gamma_{1}$ are

$$
\begin{aligned}
\hat{\mathscr{U}}^{T}(x, s ; t) & =\frac{\widehat{\Phi}_{, \tau}}{Q}-\widehat{\Psi}_{, \nu} \\
& \sim \frac{1}{c_{T}} e^{-s \nu / c_{T}} \Psi_{1}+\sum_{k=1}^{\infty}\left(e^{-s \nu / c_{L}} \frac{\Phi_{k, \tau}}{Q}+e^{-s \nu / c_{T}}\left(-\Psi_{k, \nu}+\frac{1}{c_{T}} \Psi_{k+1}\right)\right) s^{-k}
\end{aligned}
$$

and

$$
\begin{aligned}
\widehat{\mathscr{U}}^{N}(x, s ; t) & =\widehat{\boldsymbol{\Phi}}_{, \nu}+\frac{\widehat{\Psi}_{, \tau}}{Q} \\
& \sim-\frac{1}{c_{L}} e^{-s \nu / c_{L}} \Phi_{1}+\sum_{k=1}^{\infty}\left(e^{-s \nu / c_{L}}\left(\Phi_{k, \nu}-\frac{1}{c_{L}} \Phi_{k+1}\right)+e^{-s \nu / c_{T}} \frac{\Psi_{k, \tau}}{Q}\right) s^{-k} .
\end{aligned}
$$

Thus the expansion of the generalized traction on the artificial boundary is

$$
\left.\sum^{n} \alpha_{+}[\widehat{\mathscr{U}}]\right|_{\Gamma_{1}}(x, s ; t) \sim \sum_{k=-1}^{\infty} \Xi_{k}^{\alpha}\left[\left.U\right|_{\Gamma_{1}}(\cdot, t)\right](x) s^{-k} .
$$

Definitions of the tangential differential operators $\Xi_{k}^{\alpha}$, in terms of the $\Phi_{k}$ and $\Psi_{k}$, are obtained from (4.7):

$$
\begin{aligned}
\Xi_{-1}^{\alpha_{+}}\left[\left.U\right|_{\Gamma_{1}}\right]= & -\frac{\mu_{+}}{c_{T}^{2}} \Psi_{1} X^{\prime}+\frac{\lambda_{+}+2 \mu_{+}}{c_{L}^{2}} \Phi_{1} n \\
\Xi_{0}^{\alpha_{+}}\left[\left.U\right|_{\Gamma_{1}}\right]= & \left(\frac{\mu_{+}}{c_{T}} \Psi_{1, \nu}-\frac{\alpha_{+}}{c_{T}} \kappa \Psi_{1}+\left(\frac{\mu_{+}}{c_{T}}-\frac{\mu_{+}+\alpha_{+}}{c_{L}}\right) \Phi_{1, \tau}\right) X^{\prime} \\
& -\left(\frac{\lambda_{+}+2 \mu_{+}}{c_{L}} \Phi_{1, \nu}+\frac{\lambda_{+}+\mu_{+}-\alpha_{+}}{c_{L}} \kappa \Phi_{1}\right. \\
& \left.-\left(\frac{\lambda_{+}+2 \mu_{+}}{c_{L}} \frac{\mu_{+}+\alpha_{+}}{c_{T}}\right) \Psi_{1, \tau}\right) n
\end{aligned}
$$

and, for each $k \geq 1$,

$$
\begin{aligned}
\Xi_{k}^{\alpha}\left[\left.U\right|_{\Gamma_{1}}\right]= & \mu_{+}\left(\frac{c_{L}}{c_{T}} \Phi_{k, \nu \tau}-\frac{\kappa}{2} \Phi_{k, \tau}-\frac{1}{2} \Psi_{k, \nu \nu}+\left(\frac{c_{L}}{c_{T}}-\frac{1}{2}\right) \Psi_{k, \tau \tau}\right) X^{\prime} \\
& +\left(\lambda_{+}+2 \mu_{+}\right)\left(\frac{c_{T}}{c_{L}} \Psi_{k, \nu \tau}-\frac{\kappa}{2} \Phi_{k, \tau}+\frac{1}{2} \Phi_{k, \nu \nu}+\left(\frac{c_{T}}{c_{L}}-\frac{1}{2}\right) \Phi_{k, \tau \tau}\right) n .
\end{aligned}
$$

The high-frequency expansion of $\widehat{\mathscr{F}}^{\alpha}$ is determined from (4.3); truncating this expansion after a finite number of terms produces a sequence of approximate artificial boundary operators

$$
\widehat{\mathscr{F}}_{n}^{\alpha}\left[\left.\widehat{U}\right|_{\Gamma_{1}}(\cdot, s)\right]:=\sum_{k=-1}^{n} \Xi_{k}^{\alpha}\left[\left.\widehat{U}\right|_{\Gamma_{1}}(\cdot, s)\right](x) s^{-k} .
$$


All that is lacking is an explicit definition of the $\Xi_{k}^{\alpha_{+}}$in terms of $\left.U\right|_{\Gamma_{1}}$. Recurrence relations for the $\Phi_{k}$ and $\Psi_{k}$ are obtained by substituting (4.5) and (4.6) into (4.4):

$$
\begin{aligned}
& \Phi_{k+1, \nu}+\frac{\kappa}{2 Q} \Phi_{k+1}=\left\{\begin{array}{ll}
0, & k=0 \\
\frac{c_{L}}{2} \Delta \Phi_{k}, & k \geq 1
\end{array} \text { in } \Omega_{1,+},\right. \\
& \Psi_{k+1, \nu}+\frac{\kappa}{2 Q} \Psi_{k+1}=\left\{\begin{array}{ll}
0, & k=0 \\
\frac{c_{T}}{2} \Delta \Psi_{k}, & k \geq 1
\end{array} \text { in } \Omega_{1,+} .\right.
\end{aligned}
$$

If we write $U=U^{T} X^{\prime}+U^{N} n$, then the corresponding relations for the Dirichlet boundary condition (4.2) are

$$
\begin{aligned}
& \frac{1}{c_{L}} \Phi_{k+1}=\left\{\begin{array}{ll}
-U^{N}, & k=0 \\
\Phi_{k, \nu}+\Psi_{k, \tau}, & k \geq 1
\end{array} \text { on } \Gamma_{1},\right. \\
& \frac{1}{c_{T}} \Psi_{k+1}=\left\{\begin{array}{ll}
U^{T}, & k=0 \\
-\Phi_{k, \tau}+\Psi_{k, \nu}, & k \geq 1
\end{array} \text { on } \Gamma_{1} .\right.
\end{aligned}
$$

The first three artificial boundary conditions $(n=-1,0,1)$ are determined from $\Phi_{1}$ and $\Psi_{1}$. With $k=0,(4.8)$ and (4.10) yield

$$
\Phi_{1}=-c_{L} U \cdot n, \quad \Phi_{1, \nu}=\frac{c_{L}}{2} \kappa U \cdot n .
$$

The computation of tangential derivatives is straightforward.

$$
\begin{aligned}
\Phi_{1, \tau} & =-c_{L}\left(\kappa U \cdot X^{\prime}+U_{, \tau} \cdot n\right), \\
\Phi_{1, \tau \tau} & =-c_{L}\left(\left(2 \kappa U_{, \tau}+\kappa^{\prime} U\right) \cdot X^{\prime}+\left(U_{, \tau \tau}-\kappa^{2} U\right) \cdot n\right), \\
\Phi_{1, \nu \tau} & =\frac{c_{L}}{2}\left(\kappa^{2} U \cdot X^{\prime}+\left(\kappa U_{, \tau}+\kappa^{\prime} U\right) \cdot n\right),
\end{aligned}
$$

but the second normal derivative requires slightly more care:

$$
\Phi_{1, \nu \nu}=-\left.\frac{1}{2} \frac{\partial}{\partial \nu}\left(\frac{\kappa(\tau)}{Q(\tau, \nu)} \Phi_{1}\right)\right|_{\nu=0}=-\frac{3 c_{L}}{4} \kappa^{2} U \cdot n
$$

In a similar fashion $\Psi_{1}$ and its second derivatives are determined from (4.9) and (4.11).

The definitions of $\Xi_{k}^{\alpha_{+}}, k=-1,0,1$, in terms of the boundary data, that result from these calculations are

$$
\begin{aligned}
& \Xi_{-1}^{\alpha_{+}}[U]=-A_{-1}(x) U, \\
& \Xi_{0}^{\alpha_{+}}[U]=-A_{0}(x) U+B_{0}^{\alpha_{+}} U_{, \tau}, \\
& \Xi_{1}^{\alpha_{+}}[U]=A_{1}(x) U+B_{1}(x) U_{, \tau}+C_{1}(x) U_{, \tau \tau},
\end{aligned}
$$

with

$$
A_{-1}(x):=\frac{\mu_{+}}{c_{T}} X^{\prime} \otimes X^{\prime}+\frac{\lambda_{+}+2 \mu_{+}}{c_{L}} n \otimes n
$$




$$
\begin{aligned}
& A_{0}(x):=\kappa\left(\left(c_{L}-\frac{1}{2} c_{T}\right) \frac{\mu_{+}}{c_{T}} X^{\prime} \otimes X^{\prime}\right. \\
& \left.+\left(c_{T}-\frac{1}{2} c_{L}\right) \frac{\lambda_{+}+2 \mu_{+}}{c_{L}} n \otimes n\right) ; \\
& B_{0}^{\alpha_{+}}:=\left(\alpha_{+}+\frac{\mu_{+}}{c_{T}}\left(c_{T}-c_{L}\right)\right)\left(X^{\prime} \otimes n-n \otimes X^{\prime}\right) ; \\
& A_{1}(x):=\kappa^{2}\left(c_{L}-\frac{1}{2} c_{T}\right)^{2} \frac{\mu_{+}}{2 c_{T}} X^{\prime} \otimes X^{\prime} \\
& +\kappa^{\prime}\left(c_{L}-c_{T}\right)^{2} \frac{\mu_{+}}{2 c_{T}} X^{\prime} \otimes n \\
& -\kappa^{\prime}\left(c_{L}-c_{T}\right)^{2} \frac{\lambda_{+}+2 \mu_{+}}{2 c_{L}} n \otimes X^{\prime} \\
& +\kappa^{2}\left(c_{T}-\frac{1}{2} c_{L}\right)^{2} \frac{\lambda_{+}+2 \mu_{+}}{2 c_{L}} n \otimes n \\
& B_{1}(x):=\kappa\left(c_{L}-c_{T}\right)\left(\left(\frac{1}{2} c_{L}-c_{T}\right) \frac{\mu_{+}}{c_{T}} X^{\prime} \otimes n\right. \\
& \left.+\left(\frac{1}{2} c_{T}-c_{L}\right) \frac{\lambda_{+}+2 \mu_{+}}{c_{L}} n \otimes X^{\prime}\right) ; \\
& C_{1}(x):=\left(c_{L}-\frac{1}{2} c_{T}\right) \mu_{+} X^{\prime} \otimes X^{\prime} \\
& +\left(c_{T}-\frac{1}{2} c_{L}\right)\left(\lambda_{+}+2 \mu_{+}\right) n \otimes n .
\end{aligned}
$$

The final forms of the first three approximate boundary conditions, in both the frequency- and time-domains, are summarized in the following table.

$$
\begin{aligned}
& n=-1 \quad \stackrel{n}{\Sigma}^{\alpha}+[\widehat{U}]=-s A_{-1} \widehat{U} \\
& \stackrel{n}{\Sigma}^{\alpha}+[U]=-A_{-1} \dot{U} \\
& n=0 \quad \stackrel{n}{\Sigma}^{\alpha}+[\widehat{U}]=-s A_{-1} \widehat{U}-A_{0} \widehat{U}+B_{0}^{\alpha+} \widehat{U}_{, \tau}
\end{aligned}
$$

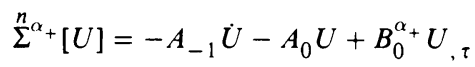

$$
\begin{aligned}
& n=1 \quad \stackrel{n}{\Sigma}^{\alpha}+[\widehat{U}]=-s A_{-1} \widehat{U}-A_{0} \widehat{U}+B^{\alpha} \widehat{U}_{, \tau} \\
& \sum^{n} \alpha_{+}[U]=-A_{-1} \ddot{U}-A_{0} \dot{U}+B_{0}^{\alpha_{+}} \dot{U}_{, \tau} \\
& +\frac{1}{s}\left(A_{1} \widehat{U}+B_{1} \widehat{U}_{, \tau}+C_{1} \widehat{U}_{, \tau \tau}\right) \\
& +A_{1} U+B_{1} U_{, \tau}+C_{1} U_{, \tau \tau}
\end{aligned}
$$

REMARK. With the exception of the first approximate artificial boundary condition $(n=-1)$, each approximate boundary condition is independent of the parameter in the sense it can be rewritten in an equivalent form which does not involve $\alpha_{+}$. For $n=0$ this follows from the fact (see Lemma 2.2) that

$$
\begin{aligned}
\Sigma^{n}[U] & =\Sigma^{n} \alpha_{+}[U]-\left(\alpha_{+}-\mu_{+}\right) M U_{, \tau} \\
& =A_{-1} \dot{U}-A_{0} U+2 \frac{\mu_{+}}{c_{T}}\left(c_{T}-\frac{1}{2} c_{L}\right) M U_{, \tau} .
\end{aligned}
$$


Since $B_{0}^{\alpha_{+}}$is the only coefficient matrix which depends upon $\alpha_{+}$, similar observations hold for all $n \geq 1$.

REMARK. The time-dependent version of the three-term boundary condition $(n=$ 1) has been differentiated with respect to time. This is done in anticipation of the variational formulation (see Sec. 4.5) as well as for notational convenience.

We conclude by noting that each approximate boundary condition is spatially local. More importantly, the temporal dependence of $\mathscr{F}_{n}^{\alpha}$ is local for $n=-1,0$ but requires the time histcry of $U$ and its first $(n+1)$ tangential derivatives for $n \geq 1$.

4.3. Dissipativity. Our investigation of the well-posedness of the artificial boundary problems proceeds exactly as in [2]. Let $\mathscr{T}^{\alpha}$ be a boundary operator of the type considered in the previous sections and let $\widehat{\mathscr{T}}^{\alpha}$ denote its Laplace transform. We consider a general class of problems that includes the exact and approximate artificial boundary problems.

Problem $4.3_{\alpha}$. Given functions $\hat{\varphi}, \hat{\psi}$, and $\hat{\chi}$, determine $\hat{U}$ which satisfies

$$
\begin{array}{cl}
\rho s^{2} \widehat{U}=\nabla \cdot \Sigma[\widehat{U}] & \text { in } \Omega \cup \Omega_{1} \\
\left.\sum^{n}{ }^{\alpha}+\hat{U}\right]=\widehat{\mathscr{T}}^{\alpha}[\widehat{U}]+\hat{\chi} & \text { on } \Gamma_{1} \\
{[\widehat{U}]=\hat{\varphi}, \quad\left[\sum^{n}[\widehat{U}]\right]=\hat{\psi}} & \text { on } \Gamma .
\end{array}
$$

Definition 4.1. The boundary operator $\mathscr{T}^{\alpha}$ is dissipative if Problem $4.3_{\alpha}$, with $\hat{\varphi}=\hat{\psi}=\hat{\chi}=0$, has no nonzero solutions for $s \in\{z \in \mathbb{C}: \operatorname{Re} z \geq 0, z \neq 0\}$.

One motivation of this definition is as follows: Suppose $\mathscr{T}^{\alpha}$ is not dissipative. Then a solution to Problem $4.3_{\alpha}$ will, in general, have poles in $\operatorname{Re} s>0$. Hence the solution to the time-dependent problem is expected to grow exponentially in time.

TheOREM 4.1. Assume $\alpha \in(-\mu, \mu]$ in $\Omega \cup \Omega_{1}$ and $\alpha_{-}-\mu_{-}=\alpha_{+}-\mu_{+}$on $\bar{\Omega}$. Sufficient conditions for the boundary operator $\mathscr{T}^{\alpha}$ to be dissipative are

$$
\begin{aligned}
& \int_{\Gamma_{1}} \widehat{\mathscr{T}}^{\alpha}[\widehat{U}] \cdot \overline{\widehat{U}} \leq 0 \text { for all } s=\xi>0 \\
& \operatorname{sgn} \operatorname{Im} \int_{\Gamma_{1}} \widehat{\mathscr{T}}^{\alpha}[\widehat{U}] \cdot \overline{\widehat{U}}=-\operatorname{sgn} \operatorname{Im} s \text { for all } s=\xi+i \eta, \\
& \text { with } \xi \geq 0, \eta \neq 0
\end{aligned}
$$

for any $\widehat{U} \not \equiv 0$ on $\Gamma_{1}$.

Proof. Let $\widehat{U}$ be a solution to Problem $4.3_{\alpha}$ with $\hat{\varphi}=\hat{\psi}=\hat{\chi}=0$. The first generalized Green identity and the continuity of $\hat{U}$ are used to show that

$$
0=s^{2} \int_{\Omega \cup \Omega_{1}} \rho|\widehat{U}|^{2}+\int_{\Omega \cup \Omega_{1}} \Sigma^{\alpha}[\widehat{U}] \cdot \nabla \overline{\widehat{U}}-\int_{\Gamma}\left[\Sigma^{n}[\widehat{U}]\right] \cdot \overline{\widehat{U}}-\int_{\Gamma_{1}} \widehat{\mathscr{T}}^{\alpha}[\widehat{U}] \cdot \overline{\widehat{U}} .
$$

Further, the interface term vanishes since, by the second hypothesis on $\alpha$ and the interface conditions, $\left[\Sigma^{n}[\widehat{U}]\right]=[\alpha-\mu] M \widehat{U}_{, \tau}=0$. The first hypothesis on $\alpha$ 
guarantees

$$
\Sigma^{\alpha}[\widehat{U}] \cdot \nabla \widehat{\widehat{U}} \geq C\left|\frac{\nabla \widehat{U}+\nabla \widehat{U}^{\top}}{2}\right|^{2} .
$$

Suppose $s=\xi>0$. Then

$$
s^{2} \int_{\Omega \cup \Omega_{1}} \rho|\widehat{U}|^{2}+\int_{\Omega \cup \Omega_{1}} \Sigma^{\alpha}[\widehat{U}] \cdot \nabla \overline{\widehat{U}} \geq C\|\widehat{U}\|_{H^{1}\left(\Omega \cup \Omega_{1}\right)}^{2}
$$

and (4.12) implies that $\|\widehat{U}\|_{H^{1}\left(\Omega \cup \Omega_{1}\right)}=0$; hence $\widehat{U}=0$ in $\Omega \cup \Omega_{1}$. (Note that this argument is not valid if $s=0$.)

When $s=\xi+i \eta$ with $\xi \geq 0$ and $\eta \neq 0$ we have

$$
0=\operatorname{Im}\left(s^{2}\right) \int_{\Omega \cup \Omega_{1}} \rho|\widehat{U}|^{2}-\operatorname{Im} \int_{\Gamma_{1}} \widehat{\mathscr{T}}^{\alpha}[\widehat{U}] \cdot \overline{\widehat{U}}
$$

If $\xi>0$, then (4.13) implies $\|\widehat{U}\|_{L^{2}\left(\Omega \cup \Omega_{1}\right)}=0$. If $\xi=0$, then $\operatorname{Im}\left(s^{2}\right)=0$ and $\operatorname{Im} \int_{\Gamma_{1}} \widehat{\mathscr{T}}^{\alpha}[\widehat{U}] \cdot \overline{\widehat{U}}=0$. But $\operatorname{sgn} \operatorname{Im} s \neq 0$ so that (4.13) implies $\widehat{U}=0$ on $\Gamma_{1}$. Then, by the uniqueness of solutions to the interface problem, we conclude that $\widehat{U}=0$ in $\Omega \cup \Omega_{1}$.

Our first application of Theorem 4.1 is to identify the parameters $\alpha$ for which $\mathscr{F}^{\alpha}$ is dissipative.

THEOREM 4.2. If $\alpha \in(-\mu, \mu]$ on $\Omega \cup \Omega_{+}$and $[\alpha-\mu]=0$ on $\Gamma_{1}$, then the exact artificial boundary operator $\mathscr{F}^{\alpha}$ is dissipative.

Proof. We provide only a sketch of the proof, the complete argument is in [21]. An energy argument on the truncated region $\Omega_{1, r}:=\left\{x \in \Omega_{1}:|x|<r\right\}$ yields

$$
0=s^{2} \rho_{+} \int_{\Omega_{1, r}}|\widehat{U}|^{2}+\int_{\Omega_{1, r}} \Sigma^{\alpha}[\widehat{U}] \cdot \nabla \overline{\widehat{U}}-\int_{\Gamma_{r}}\left[\Sigma^{n} \alpha^{\alpha}[\widehat{U}]\right] \cdot \overline{\widehat{U}}+\int_{\Gamma_{1}} \widehat{\mathscr{F}}^{\alpha}[\widehat{U}] \cdot \overline{\widehat{U}} .
$$

From the elastic radiation conditions it is determined that

$$
-\int_{\Gamma_{1}} \widehat{\mathscr{F}}^{\alpha}[\widehat{U}] \cdot \overline{\widehat{U}}=a_{2}(r) s^{2}+a_{1}(r) s+a_{0}(r)
$$

where for a sufficiently large value of $r$, say $r=r^{*}$, all three coefficients are positive.

Thus, when $s=\xi>0$,

$$
-\int_{\Gamma_{1}} \widehat{\mathscr{F}}^{\alpha}[\widehat{U}] \cdot \overline{\widehat{U}}=a_{2}\left(r^{*}\right) \xi^{2}+a_{1}\left(r^{*}\right) \xi+a_{0}\left(r^{*}\right)>0
$$

while, if $s=\xi+i \eta$ with $\xi \geq 0$ and $\eta \neq 0$,

$$
-\operatorname{Im} \int_{\Gamma_{1}} \widehat{\mathscr{F}}^{\alpha}[\widehat{U}] \cdot \overline{\widehat{U}}=\eta\left(2 \xi a_{2}\left(r^{*}\right)+a_{1}\left(r^{*}\right)\right) .
$$

Since $2 \xi a_{2}\left(r^{*}\right)+a_{1}\left(r^{*}\right)>0$, this confirms the dissipativity of $\mathscr{F}^{\alpha}$.

The primary difference between the plane and the anti-plane approximate artificial boundary conditions is the presence of the terms involving the first tangential derivative. The flexibility obtained by using the generalized traction will be exploited 
to compensate for these new terms. Thus, assuming the analogy with the anti-plane problem remains intact, we anticipate that $\mathscr{F}_{n}{ }^{\alpha}$ will be dissipative for $n=-1,0$, but not for $n=1$.

One explanation for the appearance of the additional tangential derivative terms is provided in parts (iii) and (v) of the following lemma, viz. the only skew-symmetric scalar is 0 .

LEMMA 4.1. For every $x \in \Gamma_{1}$ :

(i) $A_{-1}$ is positive definite;

(ii) $A_{0}$ is positive semi-definite if and only if $c_{T} / c_{L} \geq \frac{1}{2}$;

(iii) $B_{0}^{\alpha_{+}}$is skew-symmetric (and spatially constant) for all constants $\alpha_{+}$; moreover, $B_{0}^{\alpha_{+}}=0$ if and only if $\alpha_{+}=\alpha_{+}^{*}:=-\mu_{+}\left(1-c_{L} / c_{T}\right)$;

(iv) $A_{1}$ is positive semi-definite;

(v) $B_{1}$ is a skew-symmetric;

(vi) $C_{1}$ is positive semi-definite if only if $c_{T} / c_{L} \geq \frac{1}{2}$.

Proof. For a matrix of the form

$$
L:=a X^{\prime} \otimes X^{\prime}+b X^{\prime} \otimes n+c n \otimes X^{\prime}+d n \otimes n
$$

with scalar-valued coefficients: $\operatorname{det}(L)=a d-b c$ and $\operatorname{tr}(L)=a+b$. The physical hypotheses on the Lamé constants imply that: $c_{L}>0, c_{T}>0$, and $c_{L}-c_{T}>0$.

Since $\operatorname{det}\left(A_{-1}\right)=\left(\mu_{+} / c_{T}\right)\left(\left(\lambda_{+}+2 \mu_{+}\right) / c_{L}\right)$ and $\operatorname{tr}\left(A_{-1}\right)=\mu_{+} / c_{T}+\left(\lambda_{+}+2 \mu_{+}\right) / c_{L}$, it is immediately seen that $A_{-1}$ is positive definite. Next,

$$
\begin{gathered}
\operatorname{det}\left(A_{0}\right)=\kappa^{2} \frac{\mu_{+}}{c_{T}} \frac{\lambda_{+}+2 \mu_{+}}{c_{L}}\left(c_{L}-\frac{1}{2} c_{T}\right)\left(c_{T}-\frac{1}{2} c_{L}\right), \\
\operatorname{tr}\left(A_{0}\right)=\kappa\left(\frac{\mu_{+}}{c_{T}}\left(c_{L}-\frac{1}{2} c_{T}\right)+\frac{\lambda_{+}+2 \mu_{+}}{c_{L}}\left(c_{T}-\frac{1}{2} c_{L}\right)\right) ;
\end{gathered}
$$

thus, $A_{0}$ is positive semi-definite only when $c_{T}-\frac{1}{2} c_{L} \geq 0$. (Parts (iv) and (vi) are similar.)

The skew-symmetry of $B_{0}^{\alpha_{+}}$and $B_{1}$ is obvious. To complete the proof of (iii) note that $\left(X^{\prime} \otimes n-n \otimes X^{\prime}\right){ }_{\tau}=0$.

The positivity of $A_{-1}$ guarantees, by Theorem 4.1, the dissipativity of $\mathscr{F}_{-1}^{\alpha}$ for all admissible $\alpha$. Consider next the approximation with $n=0$. The observation that

$$
\operatorname{Im} \int_{\Gamma_{1}} A_{0} \widehat{U} \cdot \overline{\widehat{U}}=\operatorname{Im} \int_{\Gamma_{1}} B_{0}^{\alpha_{+}} \widehat{U}_{, \tau} \cdot \overline{\widehat{U}}=0
$$

implies that condition (4.13) is satisfied. Condition (4.12) requires more attention. First, $A_{0}$ is positive semi-definite if and only if $c_{T}-\frac{1}{2} c_{L} \geq 0$. Next, the skewsymmetry of $B_{0}^{\alpha_{+}}$eliminates all hope for positivity of the bilinear form for the $\widehat{U}_{, \tau}$ term. Moreover, since $\left\|\widehat{U}_{{ }_{\tau}}\right\|_{L_{2}\left(\Gamma_{1}\right)}$ cannot be bounded by $\|\widehat{U}\|_{L^{2}\left(\Gamma_{1}\right)}$, the two favorable terms do not counteract this term. The final alternative is to choose the parameter so that $B_{0}^{\alpha_{+}}=0$, i.e., $\alpha_{+}=\alpha_{+}^{*}$. To satisfy the remaining hypotheses of Theorem 4.1 let

$$
\alpha_{-}=\alpha_{-}^{*}:=\mu_{-}-\mu_{+}\left(2-\frac{c_{L}}{c_{T}}\right)
$$


and assume that

$$
\mu_{-}>\frac{\mu_{+}}{2}\left(2-\frac{c_{L}}{c_{T}}\right) \quad \text { pointwise on } \Omega \text {. }
$$

This completes the proof of the following theorem.

THEOREM 4.3. (i) $\mathscr{F}_{-1}^{\alpha}$ is dissipative for any admissible $\alpha$;

(ii) $\mathscr{F}_{0}^{\alpha}$ is dissipative if $\alpha=\alpha^{*}, c_{T} / c_{L} \geq \frac{1}{2}$, and (4.14) is satisfied.

REMARK. The physical interpretation of the restriction on the exterior wave speed is that the unbounded body cannot be too incompressible. Materials such as granite and the Earth's mantle do satisfy this constraint, but the outer core does not. In addition, the constraint on the interior shear modulus (4.14) is less restrictive than the corresponding constraint, (3.20), obtained in the analysis of the time-harmonic problem. (See also the discussion at the end of Sec. 4.4.)

The $s^{-1}$ term in the three-term approximation $(n=1)$ also has a $\widehat{U}_{\tau}$ term which is not present in the anti-plane setting. This does not present a difficulty as the second-order derivative term can be used to control the new term. The difficulty arises from the fact that both $A_{1}$ and $C_{1}$ are, in most situations, positive semi-definite. As a result, neither sufficient condition will be satisfied.

Again this parallels the anti-plane analysis. The numerical experiments in [2] indicate that exponentially growing solutions do arise from the use of the three-term approximation. These numerical results are markedly improved when a modification of the approximate operator is tested. We anticipate similar findings for the planar situation.

The idea is to consider a simple variation of $\widehat{\mathscr{F}}_{1}^{\alpha}$ which both approximates the exact artificial boundary operator $\widehat{\mathscr{F}}^{\alpha}$ as well as $\widehat{\mathscr{F}}_{1}^{\alpha}$ and improves the damping properties of the solutions. Thus we define

$$
\widehat{\mathscr{F}}_{1_{\delta}}^{\alpha}[\widehat{U}]:=-s A_{-1} \widehat{U}-A_{0} \widehat{U}+B_{0}^{\alpha+} U_{, \tau}+\frac{1}{s+\delta}\left(A_{1} \widehat{U}+B_{1} \widehat{U}_{, \tau}+C_{1} \widehat{U}_{, \tau \tau}\right),
$$

for any $\delta>0$. The corresponding boundary condition is

$$
\begin{aligned}
\sum^{n} \alpha[\dot{U}]+\delta \Sigma^{n}[U]= & -A_{-1} \ddot{U}-\left(A_{0}+\delta A_{-1}\right) \dot{U}+\left(A_{1}-\delta A_{0}\right) U \\
& +B_{0}^{\alpha_{+}} \dot{U}_{, \tau}+\left(B_{1}+\delta B_{0}^{\alpha_{+}}\right) U_{, \tau}+C_{1} U_{, \tau \tau}
\end{aligned}
$$

To investigate the dissipativity of $\widehat{\mathscr{F}}_{1_{\delta}}^{\alpha}$, let $s=\xi>0$ and consider

$$
\begin{aligned}
\int_{\Gamma_{1}} \widehat{\mathscr{F}}_{1_{\delta}}^{\alpha}[\widehat{U}] \cdot \overline{\widehat{U}}= & -s \int_{\Gamma_{1}} A_{-1} \widehat{U} \cdot \overline{\widehat{U}}-\int_{\Gamma_{1}} A_{0} \widehat{U} \cdot \overline{\widehat{U}}+\int_{\Gamma_{1}} B_{0}^{\alpha+} \widehat{U}, \tau \cdot \overline{\widehat{U}} \\
& +\frac{1}{s+\delta}\left(\int_{\Gamma_{1}} A_{1} \widehat{U} \cdot \overline{\widehat{U}}+\int_{\Gamma_{1}}\left(B_{1}-C_{1, \tau}\right) \widehat{U}_{, \tau} \cdot \overline{\widehat{U}}-\int_{\Gamma_{1}} C_{1} \widehat{U}_{, \tau} \cdot \overline{\widehat{U}}{ }_{, \tau}\right) .
\end{aligned}
$$

Recall that $A_{-1}$ is positive definite, $A_{1}$ is positive semi-definite and $B_{0}$ and $B_{1}-$ $C_{1, \tau}$ are skew-symmetric. To ensure that $A_{0}$ and $C_{1}$ are also positive definite, 
assume $c_{T} / c_{L}>\frac{1}{2}$. Then, applying Young's inequality to the skew-symmetric terms,

$$
\begin{aligned}
\int_{\Gamma_{1}} \widehat{\mathscr{F}}_{1_{\delta}}^{\alpha}[\widehat{U}] \cdot \widehat{\widehat{U}} \leq & -\left(s k_{-1}+k_{0}-c_{\alpha_{+}}\left(\epsilon_{0}\right)-\frac{k_{1}+c\left(\epsilon_{1}\right)}{s+\delta}\right)\|\widehat{U}\|_{L^{2}\left(\Gamma_{1}\right)}^{2} \\
& -\left(-\epsilon_{0}+\frac{k_{2}-\epsilon_{1}}{s+\delta}\right)\left\|\widehat{U}_{, \tau}\right\|_{L^{2}\left(\Gamma_{1}\right)}^{2}
\end{aligned}
$$

with $k_{-1}, k_{0}, k_{2}, \epsilon_{1}$, and $c\left(\epsilon_{1}\right)$ positive and $k_{1}, \epsilon_{0}$, and $c_{\alpha_{+}}\left(\epsilon_{0}\right)$ nonnegative. The goal is to demonstrate that $\alpha_{+}, \epsilon_{0}, \epsilon_{1}$, and $\delta$ can be chosen so that the right-hand side of (4.15) is nonpositive.

It is apparent that replacing $s$ with $s+\delta$ does not improve the status of the $B_{0}^{\alpha_{+}}$term; the need to have $\epsilon_{0}=c_{\alpha_{+}}\left(\epsilon_{0}\right)=0$ dictates the selection $\alpha=\alpha^{*}$. Then, choosing $\epsilon_{1}<k_{2}$ and $\delta$ sufficiently large (but independent of $\xi$ ), (4.12) will be satisfied.

The second condition (4.13) remains, in general, unfulfilled. The same situation exists in the anti-plane problem. However, numerical experiments indicate that the modified approximate boundary condition does prevent exponential growth of solutions. (We emphasize that Theorem 4.1 provides only sufficient conditions for dissipativity; necessary conditions are unknown.)

4.3. Half-space comparison. In the context of a homogeneous half-space it is a simple exercise to compare the approximate artificial boundary conditions with those obtained by Clayton and Engquist [4] and Engquist and Majda [11,12].

We consider the Dirichlet problem on $\Omega_{+}:=\left\{x \in \mathbb{R}^{2}: x_{2}>0\right\}$ and introduce an artificial boundary $\Gamma_{1}:=\left\{x \in \mathbb{R}^{2}: x_{2}=L\right\}$ with $L>0$. The first three approximate artificial boundary conditions $(n=-1,0,1)$ are

$$
\begin{gathered}
U_{, 1}+\left[\begin{array}{cc}
0 & \frac{\lambda_{+}+\mu_{+}-\alpha_{+}}{\lambda_{+}+2 \mu_{+}} \\
\frac{\alpha_{+}}{\mu_{+}} & 0
\end{array}\right] U_{, 2}+\left[\begin{array}{cc}
\frac{1}{c_{L}} & 0 \\
0 & \frac{1}{c_{T}}
\end{array}\right] \dot{U}=0 \\
U_{, 1}+\left[\begin{array}{cc}
0 & 1-\frac{c_{T}}{c_{L}} \\
\frac{c_{L}}{c_{T}}-1 & 0
\end{array}\right] U_{, 2}+\left[\begin{array}{cc}
\frac{1}{c_{L}} & 0 \\
0 & \frac{1}{c_{T}}
\end{array}\right] \dot{U}=0 \\
\dot{U}_{, 1}+\left[\begin{array}{cc}
0 & 1-\frac{c_{T}}{c_{L}} \\
\frac{c_{L}}{c_{T}}-1 & 0
\end{array}\right] \dot{U}_{, 2}+\left[\begin{array}{cc}
\frac{1}{c_{L}} & 0 \\
0 & \frac{1}{c_{T}}
\end{array}\right] \ddot{U} \\
+\left[\begin{array}{cc}
\frac{1}{2} c_{L}-c_{T} & 0 \\
0 & \frac{1}{2} c_{T}-c_{L}
\end{array}\right] U_{, 22}=0 .
\end{gathered}
$$

The three-term approximation (4.18) is exactly the same as Engquist and Majda's "modified second-order radiating boundary condition" [12, p. 336, Eq. (3.20)].

Recall that we were unable to show that (4.18) is dissipative. Even in the simplified geometry of a half-space, the well-posedness of this boundary condition is difficult to ascertain. However, numerical evidence [10] indicates that (4.18) is stable only 
for materials with $c_{T} / c_{L}>0.46$. The similarity of this finding to the theoretical restriction $c_{T} / c_{L} \geq 0.5$ needed to show that (4.17) is dissipative is intriguing, but unexplained.

4.5. Variational problems. The fact that the approximate artificial boundary conditions are natural suggests the use of finite element methods. Let $U$ be the solution to Problem $4.2_{\alpha}$ with incident wave $U^{0}$ and $\mathscr{F}_{n}^{\alpha}$ replacing $\mathscr{F}^{\alpha}$. The weak formulation is most easily discussed in terms of the total wave $W$ (see (1.1)) which satisfies

$$
\begin{array}{cl}
\rho \ddot{W}=\nabla \cdot \Sigma[W] & \text { in }\left(\Omega \cup \Omega_{1}\right) \times(0, \infty), \\
\Sigma^{n}{ }^{\alpha}[W]=\mathscr{F}_{n}^{\alpha}\left[W^{t}\right]+\mathscr{R}_{n}^{\alpha}\left[U^{0}\right] & \text { on } \Gamma_{1} \times(0, \infty), \\
{[W]=0,[\Sigma[W]]=0} & \text { on } \Gamma \times(0, \infty), \\
W(\cdot, 0)=0, \dot{W}(\cdot, 0)=0 & \text { in } \Omega \cup \Omega_{1} .
\end{array}
$$

Here $\mathscr{R}_{n}^{\alpha}\left[U^{0}\right](x, t):=\sum^{n}{ }^{\alpha}\left[U^{0}\right](x, t)-\mathscr{F}_{n}^{\alpha}\left[\left(U^{0}\right)^{t}\right](x)$.

Let $V$ be a test function on $\Omega \cup \Omega_{1}$ and assume that $\nabla\left(\alpha_{-}-\mu_{-}\right)=0$ in $\Omega$ and $[\alpha-\mu]=0$ on $\Gamma_{1}$. Then, since $\left[\Sigma^{n}+[W]\right]=0$,

$$
0=\int_{\Omega \cup \Omega_{1}} \rho \ddot{W} \cdot V+\int_{\Omega \cup \Omega_{1}} \Sigma^{\alpha}[W] \cdot V-\int_{\Gamma_{1}} \Sigma^{\alpha_{+}}[W] \cdot V
$$

and the following variational problem is obtained.

PROBlem $4.4_{\alpha, n}$. Given an incident wave $U^{0}$, find the total wave $W$ such that, for any test function $V \in H^{1}\left(\Omega \cup \Omega_{+}\right)$and all $t>0$,

$$
\begin{aligned}
& \int_{\Omega \cup \Omega_{1}}\left(\rho \ddot{W}(\cdot, t) \cdot V+\Sigma^{\alpha}[W(\cdot, t)] \cdot \nabla V\right)-\int_{\Gamma_{1}} \mathscr{F}_{n}^{\alpha}\left[W^{t}\right] \cdot V \\
& \quad=\int_{\Gamma_{1}} \mathscr{R}_{n}^{\alpha}\left[U^{0}(\cdot, t)\right] \cdot V \\
& \int_{\Omega \cup \Omega_{1}} W(\cdot, 0) \cdot V=0, \quad \int_{\Omega \cup \Omega_{1}} \dot{W}(\cdot, 0) \cdot V=0 .
\end{aligned}
$$

When the two-term approximation $(n=0)$ is used (4.20) becomes

$$
\mathscr{A}^{\alpha}(W, V)+\int_{\Omega \cup \Omega_{1}} A_{-1} \dot{W} \cdot V+\int_{\Omega \cup \Omega_{1}} \rho \ddot{W} \cdot V=\int_{\Gamma_{1}} \mathscr{R}_{0}^{\alpha}\left[U^{0}\right] \cdot V
$$

where $\mathscr{A}^{\alpha}$ is a bounded bilinear form on $H^{1}\left(\Omega \cup \Omega_{+}\right)$defined by

$$
\mathscr{A}^{\alpha}(W, V):=\int_{\Omega \cup \Omega_{1}} \Sigma^{\alpha}[W] \cdot V+\int_{\Gamma_{1}} A_{0} W \cdot V-\int_{\Gamma_{1}} B_{0}^{\alpha_{+}} W{ }_{\tau} \cdot V .
$$

Moreover, if $c_{T} / c_{L} \geq \frac{1}{2}$ and $\alpha=\alpha^{*}$, the Second Korn Inequality [9] implies that $\mathscr{A}^{\alpha^{*}}$ is coercive on $H^{1}\left(\Omega \cup \Omega_{+}\right)$.

When $W(\cdot, t)$ and $V$ are restricted to finite-dimensional subspaces of $H^{1}\left(\Omega \cup \Omega_{+}\right)$ the variational problem reduces to an initial-value problem for a system of linear 
second-order ODES in time. Thus, the finite-dimensional problems have a (unique) solution for all $t>0$. The convergence of these solutions can be investigated using "elliptic projection" techniques (see, e.g., $[1,8])$. Further results are forthcoming.

The additional damping term in the modified three-term approximation $\left(n=1_{\delta}\right)$ requires a different treatment; a mixed method seems preferable. Let $\chi(\cdot, t):=$ $\sum^{n}{ }^{\alpha}+\left.\left[W^{t}\right]\right|_{\Gamma_{1}}$ and assume $\alpha=\alpha^{*}$. The weak form of the artificial boundary condition is

$$
\begin{aligned}
\int_{\Gamma_{1}}(\dot{\chi} & \left.+A_{-1} \ddot{U}+A_{0} \dot{U}-A_{1} U\right) \cdot \eta \\
& +\delta \int_{\Gamma_{1}}\left(\chi+A_{-1} \dot{U}+A_{0} U\right) \cdot \eta \\
& -\int_{\Gamma_{1}} B_{1} U_{, \tau} \cdot \eta+\int_{\Gamma_{1}} C_{1} U_{, \tau} \cdot \eta, \tau=\int_{\Gamma_{1}} \mathscr{R}_{1_{\delta}}^{\alpha}\left[U^{0}\right] \cdot \eta
\end{aligned}
$$

where $\eta$ is a test function on $\Gamma_{1}$. Thus we seek a solution $(W, \chi)$ which satisfies (4.19), (4.22), and the initial conditions (4.21). Even though this problem is easily implemented on finite-dimensional subspaces, we have yet to succeed in proving the existence of solutions to these problems.

Acknowledgments. The author is grateful to R. C. MacCamy for many valuable discussions. Support for this work was received from the National Science Foundation under grant DMS 8601288 and from the Air Force Office of Scientific Research under grant AFOSR-88-0265.

\section{REFERENCES}

[1] G. A. Baker, Error estimates for finite element methods for second order hyperbolic equations, SIAM J. Numer. Anal. 13, 564-576 (1976)

[2] A. Barry, J. Bielak, and R. C. MacCamy, On absorbing boundary conditions for wave propagation, J. Comput. Phys. 79, 449-468 (1988)

[3] J. Bielak and R. C. MacCamy, An exterior interface problem in two-dimensional elastodynamics, Quart. Appl. Math. 41, 143-159 (1983)

[4] R. Clayton and B. Engquist, Absorbing boundary conditions for acoustic and elastic wave equations, Bull. Seismol. Soc. Amer. 67, 1529-1540 (1977)

[5] M. Costabel and E. P. Stephan, Integral equations for transmission problems in linear elasticity, J. Integral Equations Appl. 2, 221-223 (1990)

[6] M. Costabel and E. P. Stephan, On the convergence of collocation methods for boundary integral equations on polygons, Math. Comp. 49, 461-478 (1987)

[7] R. Courant and D. Hilbert, Methods of Mathematical Physics, v. II, Interscience, 1966

[8] T. DuPont, $L^{2}$-estimates for Galerkin methods for second order hyperbolic equations, SIAM J. Numer. Anal. 10, 880-889 (1973)

[9] G. Duvaut and J. L. Lions, Inequalities in Mechanics and Physics, Springer-Verlag, New YorkHeidelberg-Berlin, 1976 (translated from the French by C. W. John)

[10] S. Emerman and R. A. Stephan, Comment on "Absorbing boundary conditions for acoustic and elastic wave equations" by R. Clayton and B. Engquist, Bull. Seismol. Soc. Amer. 73, 661-665 (1983)

[11] B. Engquist and A. Majda, Absorbing boundary conditions for the numerical solution of waves, Math. Comp. 31, 639-651 (1977)

[12] B. Engquist and A. Majda, Radiation boundary conditions for acoustic and elastic wave calculations, Comm. Pure Appl. Math. 32, 313-357 (1979)

[13] D. E. Gray, ed., American Institute of Physics Handbook, McGraw-Hill, New York, NY, 1972 
[14] M. E. Gurtin, The linear theory of elasticity, Handbuch der Physik v. VIa/2, Springer-Verlag, New York-Heidelberg-Berlin, 1972

[15] G. Hellwig, Partial Differential Equations, Blaisdell Press, 1964

[16] R. L. Higdon, Numerical absorbing boundary conditions for the wave equation, Math. Comp. 49, 65-90 (1987)

[17] S. Hildebrandt and E. Wienholtz, Constructive proofs of representation theorems in separable Hilbert space, Comm. Pure Appl. Math. 17, 369-373 (1964)

[18] G. C. Hsaio and W. L. Wendland, $A$ finite element method for some integral equations of the first kind, J. Math. Anal. Appl. 58, 449-481 (1977)

[19] M. Kitahara, Boundary Integral Equation Methods in Eigenvalue Problems of Elastodynamics and Thin Plates, Elsevier, Amsterdam-New York, 1985

[20] V. D. Kupradze, Potential Methods in the Theory of Elasticity, Israel Program for Scientific Translations, 1965

[21] D. B. Meade, Interface Problems in Elastodynamics, Carnegie Mellon University, Ph. D. Thesis, 1989

[22] E. Sternberg, On the integration of the equations of motion in the classical theory of elasticity, Arch. Rational Mech. Anal. 6, 34-50 (1960) 